\title{
P-type and polarization doping of GaN in hot-wall MOCVD
}

Alexis Papamichail 



\title{
Linköping studies in science and technology
}

Licentiate Thesis No. 1929

\section{P-type and polarization doping of $\mathrm{GaN}$ in hot-wall MOCVD}

\author{
Alexis Papamichail
}

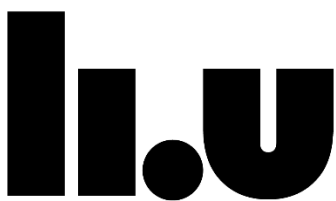

LINKÖPING UNIVERSITY

Department of Physics, Chemistry and Biology (IFM)

Linköping University, SE-581-83, Sweden

Linköping 2022 
During the course of research underlying this thesis, Alexis Papamichail was enrolled in Agora Materiae, a multidisciplinary doctoral program at Linköping University, Sweden.

(C) Alexis Papamichail, 2022

(cc) EY-NC This work is licensed under a Creative Commons AttributionNonCommercial 4.0 International License.

https://creativecommons.org/licenses/by-nc/4.0/

Printed in Sweden by LiU-Tryck, Linköping, Sweden, 2022

ISBN 978-91-7929-251-5 (print)

ISBN 978-91-7929-252-2 (PDF)

ISSN 0280-7971 


\begin{abstract}
The development of group-III nitride semiconductor technology continues to expand rapidly over the last two decades. The indium nitride $(\mathrm{InN})$, gallium nitride $(\mathrm{GaN})$ and aluminum nitride (AlN) compounds and their alloys are direct bandgap semiconductors with a wide bandgap range, spanning from infrared (IR) to deep-ultraviolet (UV), enabling their utilization in optoelectronic industry. The GaN-based light-emitting diode (LED) is already the commercial solution for efficient and energy saving lighting. Additionally, the physical properties of these materials such as the high critical electric field, the high saturation carrier velocity and the high thermal conductivity, make them promising candidates for replacing silicon ( $\mathrm{Si}$ ), and other wide-bandgap semiconductors such as silicon carbide $(\mathrm{SiC})$ in power devices. More importantly, the polarization-induced twodimensional electron gas (2DEG), forming at the interfaces of these semiconductors, led to the fabrication of the GaN-based high electron mobility transistor (HEMT). This device is suitable for high power (HP) switching, power amplifiers and high frequency (HF) applications in the millimeter-wave range up to THz frequencies. As such, HEMTs are suitable for ${ }_{5} \mathrm{G}$ communication systems, radars, satellites and a plethora of other related applications.

Achieving the efficient GaN blue LED (Nobel Prize in Physics 2014), came as a result of (partially) solving several material issues of which, $p$-type GaN was of crucial importance. Since 1992, a lot of efforts have been dedicated on the understanding and overcoming of the limitations hindering efficient $p$-type conductivity and low hole mobility in metal-organic chemical vapor deposition (MOCVD) grown $p$-GaN. The limitations arise from the fact that magnesium $(\mathrm{Mg})$ is the only efficient $p$-type dopant for GaN so far and only a very small percentage $\sim 2 \%$ of the incorporated $\mathrm{Mg}$ is active at room temperature. More limitations come from its solubility in $\mathrm{GaN}$ and the crystal quality deterioration and formation of inversion domains $\left(\mathrm{ID}_{\mathrm{s}}\right)$ at high doping levels. Free-hole concentrations in the low $10^{18} \mathrm{~cm}^{-3}$ range with mobilities at $\sim 10 \mathrm{~cm}^{2} \mathrm{~V}^{-1} \mathrm{~s}^{-1}$ demonstrate the state-of-art in MOCVD grown $p$-GaN, still leaving a wide window for improvement.

Another intensively investigated topic is related to the aluminum gallium nitride ( $\mathrm{AlGaN})$ / GaN HEMTs. High electron density and mobility of the 2DEG in the range of $10^{13} \mathrm{~cm}^{-2}$ and $\sim 2400 \mathrm{~cm}^{2} \mathrm{~V}^{-1} \mathrm{~s}^{-1}$ respectively, are reported. Interface engineering, addition of interlayers and backbarriers are only a few of the modifications introduced in the basic AlGaN/GaN HEMT structure in order to achieve the aforementioned values. Nevertheless, fundamental phenomena can still be revealed by special characterization techniques and provide a deeper understanding on the causal factors of the HEMT's macroscopic properties.
\end{abstract}

The main research results presented in this licentiate thesis are organized in three 
papers:

In paper I we report an in-depth investigation of the $\mathrm{Mg}$-doped $\mathrm{GaN}$ growth by hotwall MOCVD. We strive for exploiting any possible advantages of the hot-wall MOCVD on the growth of high-quality $p$-GaN relevant for use in HP devices. Additionally, we aim to gain a comprehensive understanding of the growth process and its limiting factors. The effects of growth conditions on the $\mathrm{Mg}$, hydrogen $(\mathrm{H})$ and carbon $(\mathrm{C})$ incorporation in $\mathrm{GaN}$ are analyzed in the framework of gallium (Ga)-supersaturation. Control of the bis(cyclopentadienyl) magnesium $\left(\mathrm{C}_{2} \mathrm{Mg}\right) /$ trimethylgallium (TMGa) ratio, the $\mathrm{V} / \mathrm{III}$ ratio and the growth temperature, resulted in high quality $p$-GaN growth on $\mathrm{AlN} / 4 \mathrm{H}-\mathrm{SiC}$ templates, showing state-of-the-art electrical properties.

In paper II, we manage to increase the free-hole concentrations in as-grown GaN:Mg in two different ways, either by growing the GaN:Mg layer on a GaN/AlN/4H-SiC template, or by modifying the gas environment of the growth. It is shown that using a $\mathrm{GaN} / \mathrm{AlN} / 4 \mathrm{H}-\mathrm{SiC}$ template results in higher carrier concentration and large improvement of the as-grown $p$-GaN resistivity. More importantly, the high amount of hydrogen $\left(\mathrm{H}_{2}\right)$ flow during GaN:Mg growth, results in higher amount of non-passivated $\mathrm{Mg}$ in the as-grown layers allowing for high free-hole concentration and significantly lower resistivity in the as-grown $p$-GaN.

Paper III focuses on the effect of aluminum (Al)-content variation in the barrier layer of AlGaN/GaN HEMTs. The THz-optical Hall effect (OHE) measurements revealed a peak of the 2DEG mobility followed by a decrease above certain value of $\mathrm{Al} \%$. We correlate this effect with the electron effective mass $\left(\mathrm{m}_{\mathrm{eff}}\right.$ ) variation and draw conclusions about the mobility limiting mechanisms. For low-Al-content AlGaN barriers, the mobility decreases because of the increase in $\mathrm{m}_{\text {eff }}$ while, in the high-Al-content barriers, the mobility is limited by the lower carrier scattering time. 


\section{POPULÄRVETENSKAPLIG SAMMANFATTNING}

Utvecklingen av halvledare baserade på grupp III-nitrider har ökat exponentiellt de två senaste årtiondena. Indiumnitrid $(\mathrm{InN})$, galliumnitrid $(\mathrm{GaN})$ och aluminiumnitrid (AlN) och deras olika legeringar besitter direkta bandgap med en oöversträffad bredd som spänner från infraröda spectrumet till det djupt ultravioletta bandet. Det i sin tur har möjliggjort en rad olika optoelektronisk tillämpningar. GaN-baserade ljusdioder (LED) är redan en kommersiellt etablerade som energieffektiva lampor. Därutöver innebär materialegenskaperna hos grupp III-nitrider, t ex ett högt kritiskt elektrisktfält, en hög mättnadsbärarhastighet samt en hög termisk ledningsförmåga, att de är goda kandidater till att ersätta kiselteknologin (Si) och andra halvledare med brett bandgap, $t$ ex kiselkarbid (SiC), i högeffektskomponenter. Mest intressant är däremot den polariseringsinducerade tvådimensionella elektrongasen (2DEG) som bildas i övergångslagren i dessa halvledare, vilket har lett till tillverkning av en GaN-baserade högelektronmobilitetstransistor (HEMT). En sådan komponent är väl anpassad för högeffektsväxling, högeffektsförstärkare samt högfrekvenstillämpningar från GHz- till THz-frekvensbanden. HEMTs har därför stor potential inom 5G-, radar- och satellitkommunikation samt en rad andra tillämpingar.

Framtagandet av den effektiva blå ljusdioden (Nobelpriset i fysik 2014) möjliggjordes bland annat genom den viktiga realiseringen av p-typ GaN. Redan 1992 påbörjades försök till att förbättra den elektriska ledningsförmågan i $p$-typ GaN- tillväxt via metallorganisk kemisk ångdeposition (MOCVD). Det har fastställts att begränsningen har legat hos doping med $\mathrm{Mg}$, som är den mest effektiva kandidaten, där endast $\sim 2 \%$ av Mg är aktivt i rumstemperatur. Fler begränsningar inkluderar inlösningskapaciteten i GaN, försämring av kristallstruktur och formation av inverteringdomäner vid höga dopingnivåer. Fria hål-koncentrationer i nivå med $10^{18} \mathrm{~cm}^{-3}$ och en mobilitet på $\sim 10 \mathrm{~cm}^{2} \mathrm{~V}^{-1} \mathrm{~s}^{-1}$ är det bästa uppnåda resultaten för MOCVD-tillväxt $p$-GaN, vilket innebär att det finns ett stort utrymme för förbättringar.

Den höga elektrontätheten och mobiliteten i 2DEG:en hos AlGaN/GaN HEMTs är ännu ett väldigt intensivt utforskat område, där respektive nivåer på $10^{13} \mathrm{~cm}^{-2} \mathrm{och}$ $\sim 2400 \mathrm{~cm}^{2} \mathrm{~V}^{-1} \mathrm{~s}^{-1}$ har rapporterats. Övergångslagerteknologier, så som införandet av nya inbäddade lager och bakbarriärer, är en del av de verktyg som har tagits fram utöver standardstrukturen för AlGaN/GaN HEMTs för att förbättra egenskaperna. Trots en mängd framsteg så upptäcks ständingt nya fundamentala processer med hjälp av ny utveckling inom karaktäriseringsteknik som bidrar till en djupare kunskap kring kasuialiteten i HEMTs.

Det vigtigaste resultaten som presenteras i denna licensiat är uppdelade i tre artiklar: I paper I demonstrerar vi en djuptgående undersökning av Mg-doping i GaN tillväxt 
med hetväggs-MOCVD där varje tänkbar variabel i reaktorsystemet har optimerats för att växa $p-\mathrm{GaN}$ av hög kvalitet mot högeffektstillämpningar. Vi presenterar en omfattande beskrivning av tillväxten dess begränsningar. Tillväxtförhållanden för $\mathrm{Mg}$-, väte- $(\mathrm{H})$, och kol- (C) inblandning betraktas som funktion av Ga-mättning. Genom att kontrollera förhållandet mellan bis-cyclopentadienylmagnesium (Cp2Mg) och trimetylgallium (TMGa), dvs V-III och tillväxttemperaturen kunde vi påvisa en $p$-GaN på AlN/4H-SiC av högkvalitet med elektriska egenskaper i toppklass.

I paper II har vi lyckat utöka fria-hålkoncentrationen i konventionellt tillväxt $\mathrm{GaN}: \mathrm{Mg}$ på två olika sätt. Antingen genom att växa GaN:Mg-lagret på en mall av GaN/AlN/4H$\mathrm{SiC}$ eller genom att modifiera gasmiljön i tillväxten. Det påvisas även att en mall av $\mathrm{GaN} / \mathrm{AlN} / 4 \mathrm{H}-\mathrm{SiC}$ resulterar i en högre laddningsbärartäthet och en förbättrad resistivitet i $p$-GaN. Mest noterbart är att ett högt H2-flöde under GaN:Mg-lagertillväxten resulterar i en större mängd icke-passiverad $\mathrm{Mg}$ i $p$-GaN vilket leder till en högre fria-hålkoncentration och en signifikant högre resistivitet.

I paper III fokuser vi på effekten av Al-koncentration på barriärlagret av AlGaN/GaN HEMTs. THz-optiska halleffekt- (OHE) mätningar visar en topp i 2DEG-mobilitet som funktion av minskad Al-halt, ner till en viss nivå. Vi korrelerar detta med elektroneffektivmassavariationer $\left(\mathrm{m}_{\mathrm{eff}}\right)$ och konkluderar att det finns begränsande mekanismer på mobiliteten. Vid låga Al-halter minskar mobiliteten pga ökad $\mathrm{m}_{\mathrm{eff}}$, medan vid höga Al-halter så begränsas mobiliteten av den lägre laddningsbärarspridningstiden. 


\section{PREFACE}

The licentiate thesis is based on the knowledge and research results accumulated during the graduate studies of Alexis Papamichail at the Swedish Center for III-Nitride Technology ( $\mathrm{C}_{3} \mathrm{NiT}$ - Janzén), in the Department of Physics, Chemistry and Biology at Linköping University from October 2018 to December 2021. The licentiate contains two main parts: the first part provides a brief introduction to the research field and a summary of the thesis work and, the second part presents the main research results summarized in three scientific papers.

The graduate studies and research were supported by Swedish Governmental Agency for Innovation Systems (VINNOVA) under the Competence Center Program Grant No. 2016 - 05190, Linköping University, Chalmers University of technology, Ericsson, Epiluvac, FMV, Gotmic, Hexagem, Hitachi Energy, On Semiconductor, Saab, SweGaN, and UMS. We further acknowledge support from the Swedish Research Council VRunder Award No. 2016 - 00889, Swedish Foundation for Strategic Research under Grants No. RIF14 - 055 and No. EM16 - 0024, and the Swedish Government Strategic Research Area in Materials Science on Functional Materials at Linköping University, Faculty Grant SFO Mat LiU No. $2009-00971$. 



\section{ACKNOWLEDGEMENT}

First and foremost I would like to express my sincere gratitude to my main supervisor Prof. Vanya Darakchieva for giving me the opportunity to join her research group as a PhD student. I always had support and the feeling of trust in me. With her positive approach, the confidence in doing things the right way, the sharing of her broad knowledge and new ideas and the continuous desire for moving beyond, she is an inspirer and great team leader at the same time. I am feeling lucky to work with you Vanya. Thank you!

Next I would like to thank my co-supervisor Assoc. Prof. Anelia KakanakovaGueorguieva for all her guidance in my research, the new ideas and the deep knowledge in the III-nitrides field that shared with me. Her advices and the long discussions on the research topics were greatly valuable to me.

I am also greatly thankful to my co-supervisor Dr. Jr-Tai Chen. He has always been supportive and a great teacher for me, sharing his tremendous experience on the MOCVD growth of III-nitride materials. With his attitude towards work and the well-structured way of thinking in problem approaching, Ted motivates me in keep trying to be a better scientist.

I am also very thankful to my friend and colleague Dr. Nerijus Armakavicius for teaching me ellipsometry and for the unlimited help.

Special thanks also to my colleague Dr. Steffen Richter for all the help and support in research topics and for being always there to help.

I am very grateful to my colleagues Dr. Philipp Kühne and Dr. Sean Knight for ellipsometry characterization and Dr. Vallery Stanishev for the electrical characterization measurements.

Prof. Mathias Schubert, thank you for all the meaningful and inspiring discussions on ellipsometry.

I would also like to thank Assoc. Prof. Plamen P. Paskov for his help and the fruitful discussions and for reviewing my papers.

I am thankful to Dr. Son Phuong Le for the samples preparation for electrical measurements and to Prof. Einar Örn Sveinbjörnsson for his patience and help on the analysis of electrical measurements.

Many thanks also to Dr. Axel Persson for his excellent work on TEM.

My gratitude also to Dr. Niklas Rorsman and Björn Hult from Chalmers University for the quick material processing and their feedback.

I am also very grateful to my colleagues Hengfang Zhang for the help and discussions on research questions, and to Dat Tran and Rosalia Delgado Carrascon for sharing their 
knowledge on common problems.

I am grateful to Dr. Pitsiri Sukkaew and Dr. Alyssa Mock for their help and support at the beginning of my PhD studies.

I am greatly thankful to Dr. Martin Eriksson for the unlimited support on Solaris maintenance. The endless hours spent with you, Ted and Sven for this purpose worth it after all.

Many thanks also to Sven Andersson for all the technical support and to Dr. Ildiko Farkas for her support and help with the experimental work environment.

I want to thank all people in the Centre for III-nitride technology $\left(\mathrm{C}_{3} \mathrm{NiT}\right.$ - Janzén) for having the chance to work with you towards our common goal.

I am also thankful to Anna Ahlgren and Camilla Höglund for the administrative support.

Many thanks also to Assistant Prof. Caroline Brommesson, Karina Malmström and Wendela Yonar for having the opportunity to be part of the Agora Materiae graduate school.

I am greatly thankful to all collaborators both in and out of LiU.

Special thanks also go to all my friends that made Linköping a nice place to live. Nerijus, Nikos, Ingemar, Laurent, Victor, Lida, Davide thank you for the unforgettable moments we spent away from work. A great thank you also to all the friends for the relaxing Friday evenings after work and the joyful events. It was great pleasure to be with you.

Lastly and more importantly, I want to express my gratitude to my family:

A great THANK YOU to my parents. Thank you for believing in me and all the selfless love and support in life. My sisters and my brother, thank you for believing in me, my love to you is far beyond words.

Chrysa, my love, you are the reason of happiness and completeness in my life. Thank you for the endless and unconditional love and support and for believing so much in me. You are the main reason for my success. Thank you! 


\section{Contents}

$\begin{array}{lc}\text { Contents } & \text { xi }\end{array}$

1 Part I 1

1.1 Introduction . . . . . . . . . . . . . . . . . 1

1.2 Properties of group-III nitride semiconductors . . . . . . . . . . . . . . 3

1.2.1 Crystal structure and properties of group-III nitrides . . . . . . . . 3

1.2.2 Polarization fields in III-nitrides and 2DEG properties . . . . . . . . 5

1.2.3 Point defects and impurities in group-III nitride materials . . . . . 10

1.2.4 Doping in III-nitrides . . . . . . . . . . . . . . . . . 11

1.2.5 Mg doping in $\mathrm{GaN}$. . . . . . . . . . . . . . . . 12

1.3 MOCVD growth of Mg-doped GaN and AlGaN/GaN HEMTs . . . . . . 16

1.3.1 MOCVD process fundamentals . . . . . . . . . . . . 17

1.3.1.1 Gas flow and growth pressure . . . . . . . . . 18

1.3.1.2 Growth temperature ............... 19

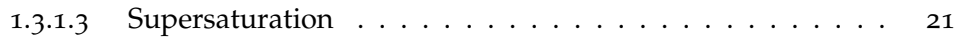

1.3.2 Hot-wall MOCVD concept ................ 22

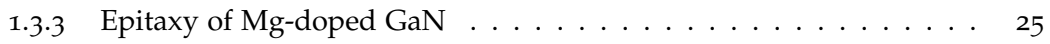

1.3.4 Epitaxy of AlGaN/GaN HEMTs . . . . . . . . . . . . . . . 28

1.4 Characterization techniques .................... 31

1.4.1 Optical Microscopy . . . . . . . . . . . . . . 31

1.4.2 Atomic force Microscopy . . . . . . . . . . . . . . 32

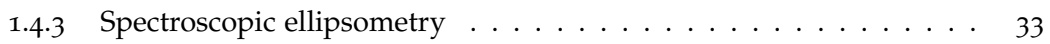

1.4.3.1 Optical Hall effect . . . . . . . . . . . . . 36

1.4.4 High-resolution X-ray Diffraction . . . . . . . . . . . . 37

1.4.4.1 Lattice parameters . . . . . . . . . . . . . 38

1.4.4.2 Dislocation density ................. 39

1.4.4.3 Al composition in $\mathrm{AlGaN}$............. 40

1.4.5 Mercury probe capacitance-voltage ............. 4 4 
1.4.6 Electrical Hall effect measurement . . . . . . . . . . . . . . . . 42

1.4.7 Contactless measurements of electrical properties . . . . . . . . . 44

1.4.8 Secondary ion mass spectroscopy . . . . . . . . . . . . 45

1.5 Summary of main results $\ldots \ldots \ldots \ldots \ldots \ldots \ldots \ldots$

$\begin{array}{ll}\text { References } & 49\end{array}$

$\begin{array}{ll}\text { List of abbreviations } & 57\end{array}$

2 Part II 61

2.1 Publications included in the thesis . . . . . . . . . . . . 61

2.2 Publications not included in the thesis . . . . . . . . . . . . 62 


\section{Part I}

\subsection{Introduction}

Group-III nitride semiconductors ${ }^{\dagger}$ continue to receive enormous interest from the academic and industrial communities. The InN, GaN and AlN compounds in combination with their alloys, are direct bandgap semiconductors and cover a wide range of bandgap energies from $\sim 0.7-6.2 \mathrm{eV}[1]$ which enables a wide range of optoelectronic device applications. Their high critical field ( $3.3 \mathrm{MV} / \mathrm{cm}$ for $\mathrm{GaN}$ and $12 \mathrm{MV} / \mathrm{cm}$ for $\mathrm{AlN}$ ), allows for very large breakdown voltage (BV). Additionally, the polar nature of III-nitrides allows for the polarization engineering at their interfaces resulting in extremely high carrier concentrations $\left(>10^{13} \mathrm{~cm}^{-2}\right)$. High carrier concentration and high mobility in combination with a high BV and high thermal conductivity, enable the realization of HP and HF device applications with outstanding performance [2-6].

HEMTs with low on-resistance values, $\mathrm{R}_{O N}<50 \mathrm{~m} \Omega$ at $30 \mathrm{~A}$, and power converters with efficiencies $>99 \%$ are already available for commercial use. Furthermore, for radio frequency (RF) amplifiers, GaN HEMTs are currently among the most suitable technologies for low-noise amplification and HF conversion in the THz-millimeter wave range. These features position III-nitride HEMTs as the cutting-edge technology for wide band and high speed communication networks, sensor devices and even space applications [7-10].

Several obstacles exist though, related to the realization of III-nitride materials epitaxy, device fabrication and product commercialization. The lack (and the high cost) of native substrates was approached by using foreign substrates, mainly sapphire, $\mathrm{Si}$ and $\mathrm{SiC}$, trading-off the lower cost with the higher dislocation densities in the hetero-epitaxial layers. Nowadays, the most commonly used technology for high-power devices is SiC-on-SiC, while tremendous efforts are dedicated on the use of GaN-on-Si [11], as the integrated

\footnotetext{
${ }^{\dagger}$ According to the IUPAC system this should be denoted as group-13 nitrides. However, because of historical reasons and widespread adoption in literature and in semiconductor community we retain the group-III notation in this thesis.
} 
circuit manufacturing industry is built based on this substrate. Achieving process compatibility on Si substrates, could bring III-nitride based devices mass production earlier to the market. Apart from the substrate cost and technology compatibility, the thermal conductivity is a crucial factor of substrate choice, especially for the HP devices. The thermal issues related to the epitaxial growth of III-nitrides are less prominent. Most of the epilayers growth is done by MOCVD and at a smaller extent by molecular beam epitaxy (MBE), with both techniques providing high quality III-nitride materials with tunable properties.

The efficient blue LED invented by Akasaki, Amano and Nakamura (Nobel prize in Physics 2014) [12] sparked intensive research and advances in III-nitrides optoelectronics. This pivotal achievement was made possible thanks to several prior steps of solving fundamental problems in epitaxial growth of III-nitrides. The development of AlN nucleation layer for accommodating the lattice mismatch between $\mathrm{GaN}$ and the sapphire substrate, the use of GaN buffer layer for reducing the dislocation density in the active layers, and the achievement of $p$-type $\mathrm{GaN}$ by thermal annealing of $\mathrm{Mg}$-doped $\mathrm{GaN}$, were fundamental contributions for the III-nitrides field and created pathways for more efficient utilization of III-nitrides unique properties.

In this thesis, we dedicate our investigations on two topics: a) p-type GaN growth via in-situ Mg-doping and $b$ ) effect of Al-content in the AlGaN barrier layer on the 2DEG properties of AlGaN/GaN HEMT structures. For the $p$-type GaN, we explore the potential benefits of the hot-wall MOCVD process on the realization of efficient $p$-GaN. Efficient $p$-type doping of $\mathrm{GaN}$ is very challenging and despite the dedicated efforts of the research community during the last almost three decades, there are still limitations at fundamental level. The free-hole concentration and mobility, and the resistivity in $p$-GaN layers are decisive factors for using the material in power devices, and thus, need to be optimized.

Concerning the AlGaN/GaN HEMTs, it is well known that variation of the Al-content in the barrier layer has direct effect on the 2DEG density and the mechanism behind this process is extensively studied. Here, we investigate the 2DEG properties using THz OHE, which in addition to charge carrier density and mobility provides information on effective mass parameters. The latter is not well understood and only scarcy investigated. We aim to better understand the basic mechanism causing the degraded mobility for high Al-content $\geq 20 \%$ in the AlGaN barrier layer of AlGaN/GaN HEMT structures in order to provide basis for further device optimization. 


\subsection{Properties of group-III nitride semiconductors}

\subsubsection{Crystal structure and properties of group-III nitrides}

III-nitride materials are direct bandgap semiconductors which make them attractive for numerous optoelectronic device applications while, the high dielectric breakdown voltage allows them to be utilized in high power devices. Their bandgap ranges from $0.7 \mathrm{eV}$ for $\mathrm{InN}$ to $3.4 \mathrm{eV}$ for $\mathrm{GaN}$, and to $6.2 \mathrm{eV}$ for AlN. Their ternary or quaternary alloys, enable the bandgap tuning (Fig. 1.1) within the whole range between $0.7-6.2 \mathrm{eV}$ and consequently, spanning from IR to deep-UV in optoelectronic and photonic devices [13, 14].

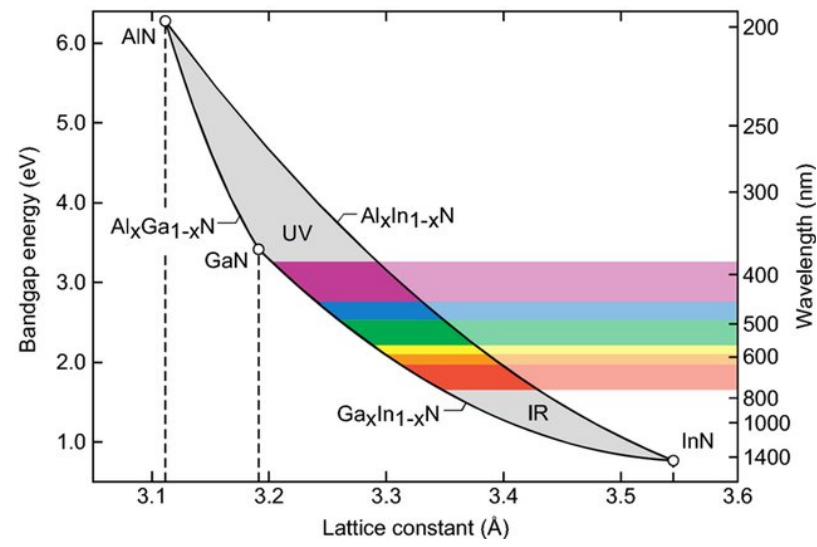

Figure 1.1: Bandgap of III-nitrides as function of their lattice constant. Reprinted from Ref. [15]

Although III-nitride semiconductors can crystallize in rocksalt, zincblende or wurtzite structure, the thermodynamically stable phase is the hexagonal wurtzite, in which the III-nitride films usually grow. The former two, rocksalt and zincblende, are meta-stable phases. The wurtzite crystal lattice consists of two hexagonal close packed (hcp) sub-lattices. One sub-lattice consists of group-III cations $\left(\mathrm{Al}^{3+}, \mathrm{Ga}^{3+}\right.$, indium $\left.(\mathrm{In})^{3+}\right)$ while the other consists of nitrogen anions $\left(\mathrm{N}^{3-}\right)$. The two sub-lattices are inter-penetrating each other and in the ideal case, they are shifted by $u c=\frac{3}{8} c$ along the [0001] direction (c-axis). $u$ is the internal parameter of wurtzite structure and corresponds to the bond distance between the anion and the cation atoms divided by the the lattice constant $c$. In the ideal wurtzite structure, the $c / a$ ratio is equal to $\frac{1}{\sqrt{u}}=\sqrt{\frac{8}{3}} \simeq 1.633$, with $a$ and $c$ being the lattice constants along the [1100] and [0001] directions respectively. As shown in Fig. 1.2, 


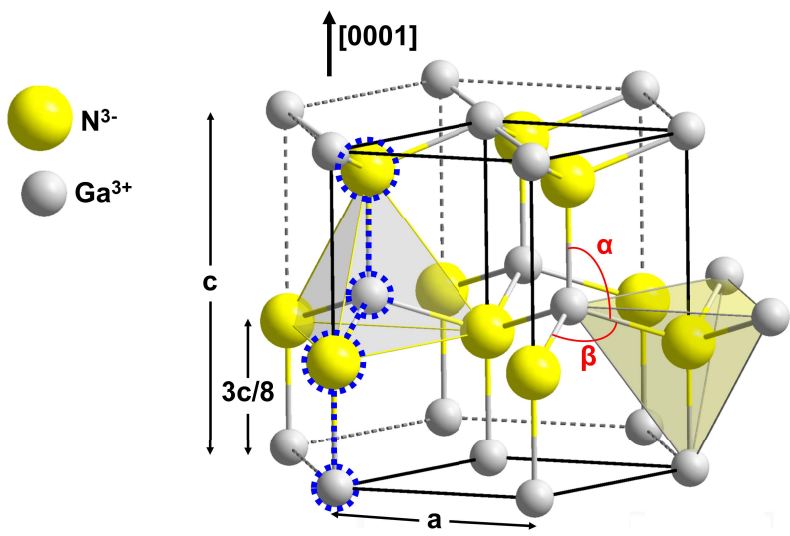

Figure 1.2: Wurtzite crystal structure of $\mathrm{GaN}$ along the [ooo1] direction. The two types of tetrahedrons ( $\mathrm{Ga}$ or $\mathrm{N}$ at the center) are shaded with the $\alpha$ and $\beta$ characteristic angles in red. The bonds and the atoms of the stacking sequence in the unit cell is marked with dashed blue lines. Modified from Wikipedia.

the wurtzite structure consists of tetrahedrons where each group-III atom is bonded with four nitrogen atoms and vice-versa. The angles $\alpha$ and $\beta$ in the ideal case are equal, $\alpha=\beta \approx 109.47^{\circ}$ [16], but in reality imperfections of the tetrahedral symmetry result in $\alpha<\beta$ and $(c / a)<\sqrt{8 / 3}$. Such non-ideality gives rise to unique properties of III-nitrides as will be described in section 1.2.2.

Typically, the wurtzite III-nitride materials grow along the $c$-axis but the wurtzite lattice lacks inversion symmetry along this axis, meaning that upon inversion, the group-III atoms will become nitrogen atoms and vice-versa. Consequently, two variations of the growth direction can be recognised: the metal-polar and the nitrogen-polar direction, designated as (Al-, Ga- or In-) polar and nitrogen (N)-polar respectively. Ga-polar GaN grows along the [0001] direction while N-polar GaN grows along the [0001] direction as shown in Fig. 1.3. As can be seen from this schematic, the GaN polarity is determined by the direction of tetrahedrons in the unit cell. When three of the bonds of a Ga atom in a tetrahedral are pointing towards the substrate, the material is then determined as Ga-polar. In the opposite case where the three bonds of the Ga atom are pointing at the growth direction, the material is defined as N-polar. It should be noted that the surface termination atoms could be either $\mathrm{Ga}$ or $\mathrm{N}$ in both metal- or nitrogen-polar GaN although, it is common to use the terms Ga- or N-terminated and Ga- or N-face as sign of the GaN polarity. 

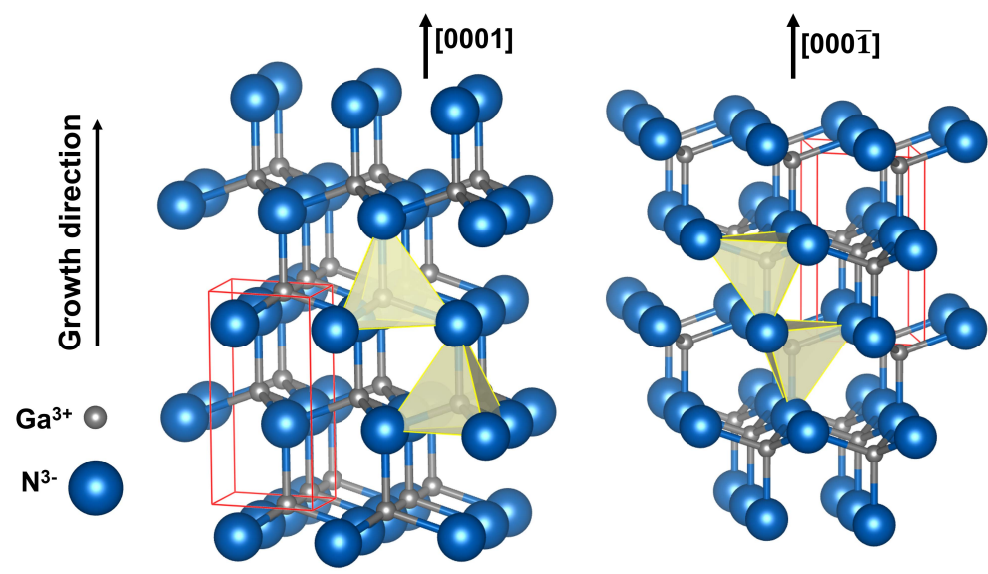

Figure 1.3: Schematic of wurtzite GaN crystal lattice showing the polarity along the growth direction. Left: Ga-polar GaN grown along the [0001] direction and, right: N-polar GaN grown along the [0001] direction. Small grey spheres represent the Ga-cations and the large blue spheres represent the $\mathrm{N}$-anions. The unit cell is sketched by solid lines.

\subsubsection{Polarization fields in III-nitrides and 2DEG properties}

The electronegativity difference between the nitrogen and the group-III atom in wurtzite III-nitrides results in small ionicity degree in the group-III - N covalent bond. Since the electronegativity of $\mathrm{N}$ is higher than that of group-III atoms, the electron cloud of the covalent bond is shifted closer to the $\mathrm{N}$ atom thus, creating an electric dipole. Despite that the electric dipoles in the ideal wurtzite III-nitrides would cancel-out within the tetrahedral building blocks, both in the in-plane and out-of-plane direction (c-axis), the non-ideality of the tetrahedrons' structure in wurtzite III-nitrides (bonding angle $\alpha<\beta \Leftrightarrow(c / a)<\sqrt{8 / 3}$ ) results in not fully compensated electric dipoles along the $c$-axis. Thus, a polarization is present in the tetrahedrons pointing from the $\mathrm{N}$ atom to the group-III atom.

The accumulated macroscopic polarization field along the $c$-axis is called spontaneous polarization $\left(\mathrm{P}_{\mathrm{SP}}\right)$ because it occurs at strain-free conditions. The $\vec{P}_{\mathrm{SP}}$ direction points towards the substrate for metal-polar material and its magnitude increases from $\mathrm{GaN}$ to $\mathrm{InN}$ to $\mathrm{AlN}$ along with decreasing $c_{0} / a_{0}$ as shown in Table 1.1. Inside the material, the dipole charges are screened by the nearest neighbouring dipole charges with the opposite sign. Since the wurtzite lattice lacks inversion symmetry, there are remaining unscreened charges at the 
Table 1.1: Material properties of wurtzite AlN, GaN, and InN.

\begin{tabular}{lccc}
\hline & $\mathrm{AlN}^{a, b, c}$ & $\mathrm{GaN}^{a, b, d, e}$ & $\mathrm{InN}^{a, b, e}$ \\
\hline Bandgap $(\mathrm{eV})$ & 6.2 & 3.4 & 0.64 \\
\hline Lattice constants @300 K $(\AA)$ & & & \\
$a_{0}$ & 3.1120 & 3.1893 & 3.5330 \\
$c_{0}$ & 4.9820 & 5.1852 & 5.6930 \\
$c_{0} / a_{0}($ ideally $\approx 1.633)$ & $\approx 1.601$ & $\approx 1.626$ & $\approx 1.609$ \\
\hline$P_{\mathrm{SP}}[0001]\left(\mathrm{C} / \mathrm{m}^{2}\right)$ & -0.081 & -0.029 & -0.032 \\
\hline Piezoelectric coefficients $\left(\mathrm{C} / \mathrm{m}^{2}\right)$ & & & \\
$e_{31}$ & -0.60 & -0.49 & -0.57 \\
$e_{33}$ & 1.46 & 0.73 & 0.97 \\
\hline Elastic coefficients $(\mathrm{GPa})$ & & & \\
$C_{13}$ & 107 & 101 & 85 \\
$C_{33}$ & 404 & 395 & 236 \\
\hline
\end{tabular}

metal- and $\mathrm{N}$-face (negative and positive respectively) of the crystal boundaries which are called polarization induced sheet charges. This explains why $\mathrm{P}_{\mathrm{SP}}$ is measured in charge per unit area $\left(\mathrm{C} / \mathrm{m}^{2}\right)$ units.

The biaxial strain induced in the wurtzite crystal by the applied stress, may further deform the tetrahedrons. This implies formation of an additional polarization field along the $c$-axis of the crystal. If the in-plane lattice parameter $(a)$ of the epilayer is smaller than the substrate lattice parameter, then the epilayer is under biaxial tensile strain and the bond angle $\alpha$ becomes even smaller. Consequently, the induced polarization field is parallel to the $c$-axis and points to the same direction as the $\mathrm{P}_{\mathrm{SP}}$. On the contrary, when the in-plane lattice parameter of the epilayer is larger than the substrate lattice parameter, the epilayer is under compressive strain and the bond angle $\alpha$ becomes larger. As a result, the induced polarization field is anti-parallel to $\mathrm{P}_{\mathrm{SP}}$. The additional polarization field described above is called piezoelectric polarization $\left(\mathrm{P}_{\mathrm{PE}}\right.$ ) and can be calculated by Eq. 1.1 while the polarization related parameters in III-nitrides are listed in Table 1.1. The total polarization in a III-nitride layer (i.e. the sum of $\mathrm{P}_{\mathrm{SP}}$ and $\mathrm{P}_{\mathrm{PE}}$ ) Eq.1.2 is a very useful material property utilized for the 2DEG formation as will be discussed later on.

$$
\begin{array}{r}
P_{\mathrm{PE}}=\left(\epsilon_{\mathrm{xx}}+\epsilon_{\mathrm{yy}}\right) e_{31}+\epsilon_{\mathrm{zz}} e_{33}=2 \epsilon_{\mathrm{xx}} e_{31}+\epsilon_{\mathrm{zz}} e_{33} \\
P_{\mathrm{PE}}=2\left(\frac{\alpha-\alpha_{0}}{\alpha_{0}}\right)\left(e_{31}-\frac{C_{13}}{C_{33}} e_{33}\right),
\end{array}
$$

where $\epsilon_{\mathrm{xx}}=\epsilon_{\mathrm{yy}}$ and $\epsilon_{\mathrm{zz}}$ are the in-plane and out-of-plane strain components respectively, 
$e_{31}$ and $e_{33}$ are the piezoelectric coefficients and $C_{13}$ and $C_{33}$ the elastic coefficients.

$$
\vec{P}_{\text {tot. }}=\vec{P}_{\mathrm{SP}}+\vec{P}_{\mathrm{PE}}
$$

Polarization induced sheet charges appear on the crystal boundaries along the $c$-axis of the wurtzite crystal. For metal-polar material, negative sheet charge appears at the top side and positive sheet charge at the bottom side (towards substrate) of the crystal. When combining two layers of different III-nitride materials e.g. AlGaN on top of GaN, the polarization induced sheet charge at their interface will be the sum of the sheet charges at the two sides of the interface. In other words, the interface charge will be the sum of the positive sheet charge from the AlGaN bottom surface and the negative sheet charge from the GaN top surface. If the total charge density at the interface is positive $(+\sigma)$, then free electrons will be accumulated in order to compensate the polarization induced positive charge $+\sigma$. Thus, a $2 \mathrm{DEG}$ is formed with a sheet electron density $n_{\mathrm{s}}$ only if the conduction band of the formed triangular quantum well at the interface drops below the Fermi level $\left(\mathrm{E}_{\mathrm{F}}\right)[22,23]$ as depicted schematically in Fig. 1.4.

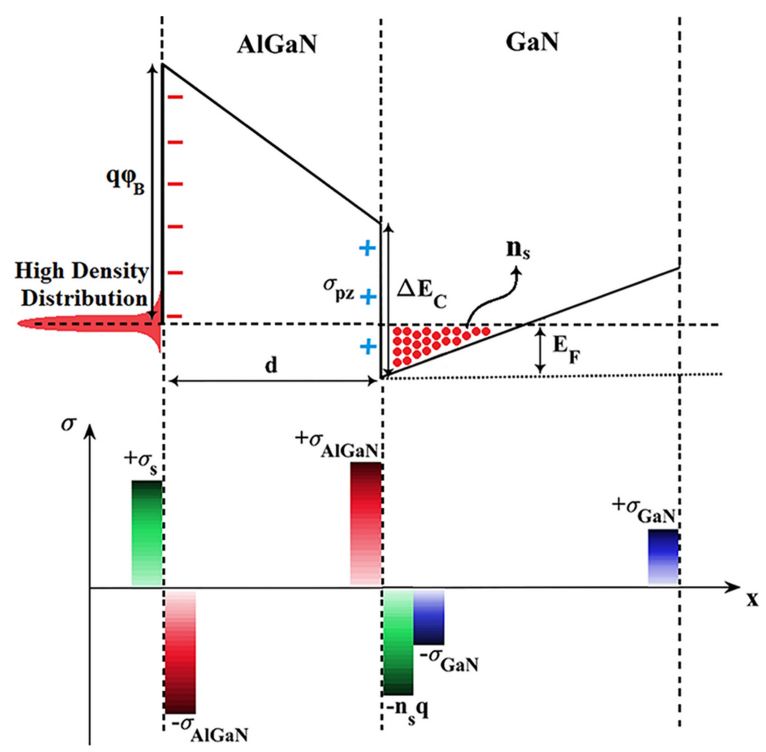

Figure 1.4: Energy band diagram of the commonly used AlGaN/GaN heterostructure (top) and the charge distribution at the interfaces with $n_{\mathrm{S}}$ being the electron density of the 2DEG (bottom). Reprinted from Ref. [24]. 
The 2DEG is positioned at the lower AlGaN/GaN interface as shown in Fig. 1.5. Similarly, if the total charge density at the interface is negative $(-\sigma)$, a two-dimensional hole gas (2DHG) is expected to form in order to compensate the fixed negative charge at the interface, only if the valence band edge of the bilayer is shifted above the $\mathrm{E}_{\mathrm{F}}$. The
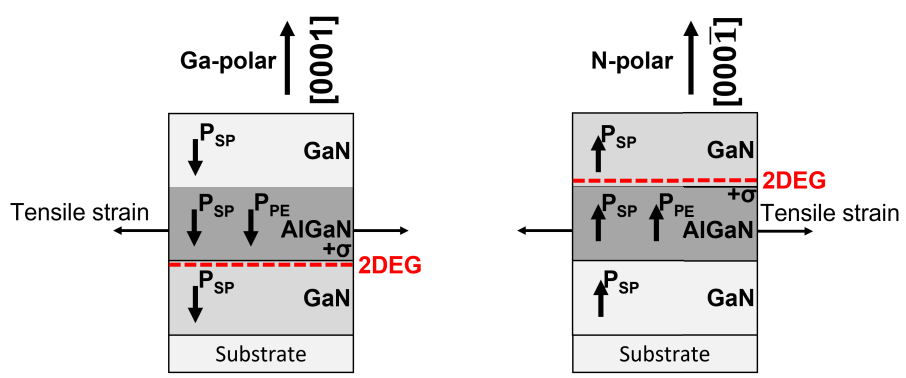

Figure 1.5: Metal- and N-polar GaN/AlGaN heterostructures. The spontaneous and piezoelectric polarization along the $c$-axis, $\mathrm{P}_{\mathrm{SP}}$ and $\mathrm{P}_{\mathrm{PE}}$ respectively, and the polarization induced charge density $\sigma$ at the heterojunction. The structures are representing Ga-polar and N-polar HEMTs.

2DEG density is affected by several factors in an AlGaN/GaN heterostructure, like the Al-composition and the thickness of the barrier AlGaN layer and the addition of a GaN cap layer. Increasing the $\mathrm{Al}$-composition in the $\mathrm{AlGaN}$ layer results in higher $\mathrm{P}_{\mathrm{SP}}$ and $\mathrm{P}_{\mathrm{PE}}$ and hence, increase in the 2DEG density. Additionally, a thicker AlGaN barrier will result in higher $n_{\mathrm{S}}$ while, the opposite effect will be present with increasing the thickness of the GaN cap layer according to Eq.1.3 [25].

$$
N_{\mathrm{S}}=\frac{1}{1+\frac{d_{\text {cap GaN }}}{d_{\text {barrier AlGaN }}}}\left[\frac{\sigma^{\text {pol }}}{q}-\frac{\varepsilon_{r, A l G a N} \varepsilon_{0}}{d_{\text {barrier AlGaN }}}\left(q \varphi_{\mathrm{B}}+\mathrm{E}_{\mathrm{F}}\right)\right],
$$

where: $d_{\text {cap GaN }}$ and $d_{\text {barrier AlGaN }}$ are the thicknesses of cap GaN and barrier AlGaN layers while, $\sigma^{\mathrm{pol}}, q, \varepsilon_{r, A l G a N}, \varepsilon_{0}$ and $q \varphi_{\mathrm{B}}$ is the polarization-induced charge density, the elemental electron charge, the dielectric constant of the barrier layer, the vacuum permittivity and the surface barrier height, respectively. The role of each of the aforementioned layers in the HEMT structure will be discussed in detail in section 1.3.4.

The transport properties of the 2DEG are affected by several factors. In general, high 2DEG mobility values can be achieved using the low-impurity GaN and low-roughness of the AlGaN/GaN interface [26, 27]. Nevertheless, the scattering mechanisms that affect mobility in $\mathrm{AlGaN} / \mathrm{GaN}$ heterostructures must be taken into consideration for explaining 
the decrease in mobility and for tuning of the structure parameters according to the desired outcome. Briefly, these mechanisms include: scattering of the 2DEG by acoustic and optical phonons (the effect is prominent at room temperature), alloy disorder scattering (increases with the Al-content in the barrier), remote ionized and background impurities scattering and finally, scattering by dislocations. All these mechanisms, specifically for the AlGaN/GaN system are extensively discussed by Jena et al. in Ref. [27].

1. The scattering by phonons is the main mechanism limiting the 2DEG mobility at room temperature in the range of $\sim 2000-2400 \mathrm{~cm}^{2} \mathrm{~V}^{-1} \mathrm{~s}^{-1}$. The effect becomes more pronounced for high 2DEG densities $\left(10^{13} \mathrm{~cm}^{-2}\right)$.

2. The alloy disorder scattering is present at the AlGaN/GaN interface and gets more pronounced at high 2DEG densities leading to a decrease of mobility. With increasing 2DEG density, the 2DEG wavefunction penetrates more into the barrier because it is shifted closer to the junction. Similarly, for low Al-composition barrier, the conduction band discontinuity is smaller leading to large penetration of the 2DEG wavefunction in the barrier. Again, the increased alloy scattering results in lower mobility. Introducing a very thin $(\sim 1 \mathrm{~nm}$ AlN interlayer between the GaN channel and the AlGaN barrier effectively diminishes the penetration of the 2DEG wavefunction in the AlGaN and effectively minimize the alloy scattering in the process [28, 29].

3. The $\mathrm{AlGaN} / \mathrm{GaN}$ interface roughness is introducing another scattering mechanism for the 2DEG, although the mobility dependence on the interface roughness is scaling with a factor of $L^{-6}$ where $L$ is the thickness of the quantum well [3o]. Nevertheless, the growth processes nowadays allow for the formation of atomically smooth interfaces rendering mobilities $>2000 \mathrm{~cm}^{2} \mathrm{~V}^{-1} \mathrm{~s}^{-1}[31]$.

4. Ionized impurities are always present in a heterojunction but they could give notable scattering effects only if the barrier thickness is less than $\sim 10 \mathrm{~nm}$. At the same time, the effects are more pronounced at low 2DEG densities. The background impurities, which are usually donor-like $\left(\mathrm{Si}, \mathrm{O}\right.$ and $\mathrm{V}_{\mathrm{N}}$ ), have typical concentrations in the $\sim 10^{16} \mathrm{~cm}^{-3}$ range, and the scattering effect is very weak. For low 2DEG density, impurity concentration of $\sim 10^{18} \mathrm{~cm}^{-3}$ would negatively affect the mobility. For high 2DEG density, the mobility increases and it is not prominently affected by the background impurities.

5. Threading dislocations in wurtzite $c$-oriented III-nitrides are introduced along the $c$-axis thus, intervening perpendicular to the $2 \mathrm{DEG}$ and affecting its transport properties. The dangling bonds created along a threading dislocation, introduce additional states in the energy gap and the dislocations act like carrier traps. On device level the 
dislocations consist current leakage paths. For dislocation densities in the common $10^{8} \mathrm{~cm}^{-2}$ range, the mobility of low density (low $10^{12} \mathrm{~cm}^{-2}$ ) 2DEGs is affected more by this scattering mechanism. Of course, high dislocation densities severely affect the mobility.

\subsubsection{Point defects and impurities in group-III nitride materials}

The low intrinsic carrier concentration in wide bandgap semiconductors, like GaN and AlN, turn them into insulators at room temperature. The carrier concentration of a semiconductor is affected by the effective density of states in the conduction and valence bands $\left(N_{\mathrm{C}}\right.$ and $N_{\mathrm{V}}$ ), the semiconductor bandgap $\mathrm{E}_{g}$ and the temperature:

$$
n_{i}=\sqrt{N_{\mathrm{C}} N_{\mathrm{v}}} e^{\frac{-E_{g}}{2 k T}}
$$

where $k$ is the Boltzmann constant. Nevertheless, in reality native defects in the crystal lattice and incorporation of impurity atoms during growth of unintentionally doped (UID-) material have a strong impact on the conductivity of the semiconductor. These defects and impurities introduce additional energy states in the bandgap, forming donors or acceptors of electrons. When they are ionized, the former provide electrons to the conduction band turning the semiconductor to $n$-type, while the latter capture electrons from the valence band creating a hole concentration there and consequently turning the semiconductor to $p$-type. If the dopants' ionization energy is relatively low (shallow dopants) comparable to the thermal energy at room temperature $(\approx 25 \mathrm{meV})$, then high free electron or hole concentrations are present at room temperature.

The conductivity of wide bandgap semiconductors is widely affected by impurities while, the intentional doping is compromised by intrinsic defects of electrically active sites of the opposite carrier species. These electrically active traps may either be shallow, leading to strong compensation of the intentional dopants, or deep, resulting in a smaller compensation effect. Defects of such type are the vacancies $\left(\mathrm{V}_{\mathrm{N}}\right.$ or $\left.\mathrm{V}_{\mathrm{Ga}}\right)$, the antisites $\left(\mathrm{N}_{\mathrm{Ga}}\right.$ or $\left.\mathrm{Ga}_{\mathrm{N}}\right)$ and the interstitials $\left(\mathrm{Ga}_{\mathrm{i}}\right.$ or $\left.\mathrm{N}_{\mathrm{i}}\right)$. Among them, $\mathrm{V}_{\mathrm{N}}$ is a common defect with mid-to-shallow donor characteristics $(90-260 \mathrm{meV}$ below the conduction band edge) while $\mathrm{V}_{\mathrm{Ga}}$ is also abundant in $\mathrm{GaN}$ due to its low formation energy [32].

UID-GaN can easily be $n$-type due to oxygen $(\mathrm{O})$ and $\mathrm{Si}$ impurities incorporation during the MOCVD growth. $\mathrm{O}$ and $\mathrm{Si}$ are shallow donors in GaN. Although nitrogen vacancy $\left(\mathrm{V}_{\mathrm{N}}\right)$ also causes $n$-type conductivity, the formation energy of this defect is rather high [33] under normal growth conditions, limiting its contribution to $n$-type conductivity in 
as-grown $\mathrm{GaN}$. On the contrary, $\mathrm{Si}$ is present during growth originating from the quartz tube chamber while, $\mathrm{O}$ mainly originates from water adsorbed on the chamber walls during the exposure of chamber to the atmosphere for substrate loading. Thus, pumping the cell down to low $10^{-5}$ mbar before growth is very important. Other sources of $\mathrm{O}$ are the carrier gases $\left(\mathrm{N}_{2}\right.$ and $\left.\mathrm{H}_{2}\right)$ and the precursors (metal-organic and $\left.\mathrm{NH}_{3}\right)$. In order to reduce the $\mathrm{O}$, ultra-high purity metal-organic sources are used while the $\mathrm{NH}_{3}$ and the carrier gases are purified before use.

$\mathrm{C}$ is amphoteric in $\mathrm{GaN}$ and although when is substitutional to $\mathrm{Ga}$ site $\left(\mathrm{C}_{\mathrm{Ga}}\right)$ acts like a shallow donor, the as-grown $\mathrm{GaN}$ epilayers with high $\mathrm{C}$-levels are highly resistive. For this reason, $\mathrm{C}$ is used for rendering insulating properties of $\mathrm{GaN}$. The deep acceptor-like behavior of $C$ is obtained when it is in nitrogen substitutional site $\left(C_{N}\right)$ [34]. Another way of achieving insulating properties is the intentional iron $(\mathrm{Fe})$ doping which creates a deep acceptor level in $\mathrm{GaN}$. Either way for rendering semi-insulating $\mathrm{GaN}$ is challenging. On the one hand, $\mathrm{C}$ is abundant in MOCVD processes due to the cracking of metal-organic precursors and its incorporation in $\mathrm{GaN}$ is highly sensitive to the growth conditions e.g. temperature and pressure. On the other hand, intentional Fe-doping has the challenge of sharp-profile growth due to memory effects resulting in difficult control of the doping profile.

The UID-GaN samples in this work were semi-insulating while both the UID- and Mgdoped GaN epilayers had $\mathrm{O}$ and Si levels close to the detection limits of SIMS $(\sim 3-$ $5 \times 10^{15} \mathrm{~cm}^{-3}$ ) and C-levels at $\sim 5 \times 10^{15}-1 \times 10^{16} \mathrm{~cm}^{-3}$. These conditions set a good reference point for working with the intentional Mg-doping of $\mathrm{GaN}$.

\subsubsection{Doping in III-nitrides}

Doping in III-nitrides is achieved via substitution of group-III or group-V atoms by group-II or group-IV atoms for $p$ - or $n$-type doping, respectively. $\mathrm{Si}$, germanium (Ge) and selenium (Se) act as donors in $\mathrm{GaN}$ when substituting $\mathrm{Ga}\left(\mathrm{Si}_{\mathrm{Ga}}, \mathrm{Ge}_{\mathrm{Ga}}\right.$ and $\left.\mathrm{Se}_{\mathrm{Ga}}\right)$. Additionally, $\mathrm{O}$ is a donor when incorporated on the $\mathrm{N}$ site $\left(\mathrm{O}_{\mathrm{N}}\right)$. All of these donors have ionization energy $<30 \mathrm{meV}$ which is comparable to the thermal energy at room temperature and therefore, almost all of the incorporated dopant atoms are ionized at these conditions. Considering the aforementioned donors, $\mathrm{Si}$ is the dopant of choice for $n$-type doping. $\mathrm{O}$ is not desired in the MOCVD process, while it is difficult for Ge and Se to incorporate in the $\mathrm{GaN}$ lattice because of their large size compared to $\mathrm{Si}$. C has amphoteric character but results in highly resistive $\mathrm{GaN}$ as discussed before. The group-II atoms suitable for $p$-type doping in III-nitrides are beryllium (Be) and $\mathrm{Mg}$ with the latter being the only successful $p$-type dopant for GaN and AlGaN. Mg is the shallowest acceptor for GaN but its ionization energy is relatively high $(\sim 200 \mathrm{meV})$ while for AlN is $\sim 650 \mathrm{meV}$. 
Since our study relates to $\mathrm{Mg}$ doping in $\mathrm{GaN}$, the issues and limitations related to the $\mathrm{Mg}$ doping in $\mathrm{GaN}$ are discussed in detail in section 1.2.5.

\subsubsection{Mg doping in $\mathrm{GaN}$}

1. Solubility

The solubility of $\mathrm{Mg}$ dopant in $\mathrm{GaN}$ represents the ability (maximum concentration) of $\mathrm{Mg}$ atoms to be incorporated in the $\mathrm{GaN}$ lattice under thermodynamic equilibrium conditions. As the dopant atom replaces a matrix atom, the dopant solubility depends on the size difference and chemical similarity between the two types of atoms, the temperature, and the abundance of dopant and matrix atoms in the growth environment. These differences in size and chemical similarity lead to substitution of dopants at the wrong lattice sites while, exceeding the solubility limit would lead to dopant atoms segregation or formation of other phases. In the hcp unit cell of $\mathrm{GaN}$, the maximum number of Ga- (or N-) sites available for $\mathrm{Mg}$ substitution, is given by Eq. 1.5.

$$
N_{\text {available sites }}=\frac{n_{\mathrm{GaN}}}{V_{\mathrm{GaN}}}=\frac{6}{\frac{3 \sqrt{3} a^{2} c}{2}} \approx 4.4 \times 10^{22} \mathrm{~cm}^{-3},
$$

where $\mathrm{n}_{\mathrm{GaN}}$ is the number of atoms in the unit cell, $\mathrm{V}_{\mathrm{GaN}}$ is the volume of the unit cell and $a$ and $c$ are the strain-free lattice constants of GaN [20]. Therefore, there is a limitation in $\mathrm{Mg}$ doping in GaN before alloying which is $\sim 10^{20}-10^{21} \mathrm{~cm}^{-3}$.

For $\mathrm{Mg}$ doping in $\mathrm{GaN}$, the formation of $\mathrm{Mg}_{3} \mathrm{~N}_{2}$ phase is the limiting factor for $\mathrm{Mg}$ solubility. The lowest formation energy of $\mathrm{Mg}_{3} \mathrm{~N}_{2}$ phase and consequently the $\mathrm{Mg}$ solubility limit in $\mathrm{GaN}$ (maximum $\mathrm{Mg}_{\mathrm{Ga}}$ concentration) happens for $\mathrm{GaN}$ growth under $\mathrm{N}$-rich conditions [35]. It has been reported that the the maximum $\mathrm{Mg}$ concentration in GaN before reaching the saturation limit is in the mid- $10^{19} \mathrm{~cm}^{-3}$ range [36].

\section{High ionization energy}

The ionization energy of a donor (or an acceptor) denotes the amount of energy needed to move an electron from the donor atom to the conduction band (or to move a hole from the acceptor atom to the valence band i.e. move an electron from the valence band to the acceptor atom). In case that the ionization energy is smaller than the thermal energy, $\mathrm{kT}$, at room temperature $(\approx 25 \mathrm{meV})$, the dopants are completely ionized. Otherwise, the dopants are electrically inactive even if they are incorporated at the right crystal lattice site. Considering the hydrogen atom approximation (which is valid for shallow "effective mass" acceptors) [37], the Mg ionization energy in GaN 
can be estimated by Eq. 1.6:

$$
E_{\mathrm{A}} \approx \frac{13.6}{\varepsilon_{\mathrm{r}}^{2}} \frac{m_{\mathrm{h}}^{*}}{m_{0}}
$$

where $\frac{m_{\mathrm{h}}^{*}}{m_{0}}$ is the hole effective mass in GaN $\left(1.1-1.3\right.$ [38]) and $\varepsilon_{\mathrm{r}}$ is the dielectric constant of GaN (8.9). This means that only a very small amount of acceptors $(\sim 1 \%)$ are electrically active at room temperature. In order to get $100 \%$ ionized acceptors, the temperature should be in the range of $2000^{\circ} \mathrm{C}$. Despite that this approximation gives reasonable values for $\mathrm{Mg}$ activation energy in $\mathrm{GaN}$, more recent approaches, classified Mg as "polaronic deep acceptor" [34, 39] with activation energy $\sim 180 \mathrm{meV}$. On the contrary, the ionization energy of $\mathrm{Si}$ and $\mathrm{O}$ donors in $\mathrm{GaN}$ is in the range of $\sim 30 \mathrm{meV}$ thus, they are almost fully ionized under normal conditions as shown in Fig. 1.6. As a result, much higher $\mathrm{Mg}$ concentrations should be incorporated in $\mathrm{GaN}$ in order to overcome the unintentional donor concentration, typically low $10^{16} \mathrm{~cm}^{-3}$, and the low activation. However, increasing the $\mathrm{Mg}$ concentration above mid- $10^{19} \mathrm{~cm}^{-3}$ results in structural defects which deteriorate the electrical properties progressively.

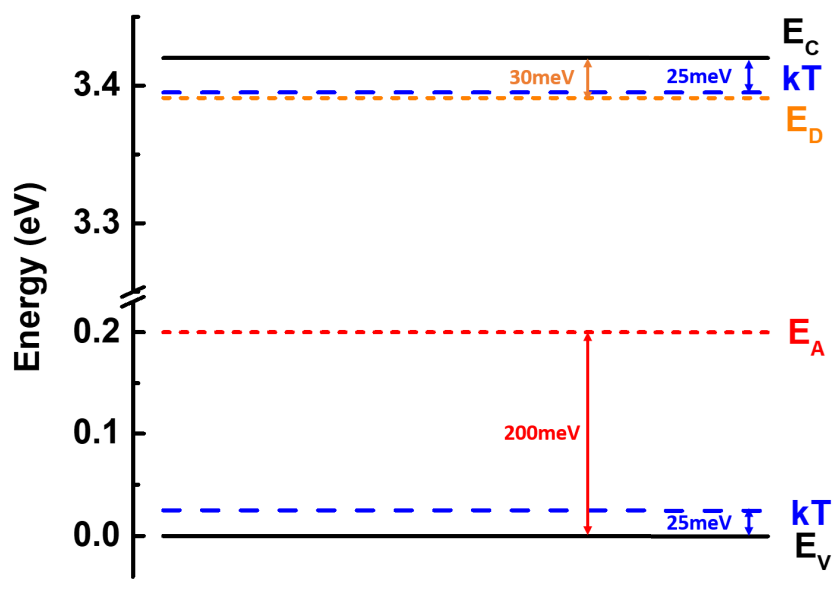

Figure 1.6: Energy levels of Si-donors and Mg-acceptors in GaN compared to the thermal energy at RT.

3. Surface segregation and pyramidal inversion domains (PIDs) 
$\mathrm{Mg}$ atoms tend to accumulate at the GaN surface. In these areas, where the $\mathrm{Mg}$ forms $\sim 1$ monolayer, the high concentration exceeds the solubility limit and the $\mathrm{Mg}$ atoms form defects, in order to deplete the material excess, which causes polarity inversion [40-42]. In this case, hexagonal-based pyramids are formed because of the preferential GaN growth towards specific directions which happens at high $\mathrm{Mg}$ concentrations [43]. When the Mg levels overcome the solubility limit, formation of one monolayer of $\mathrm{Mg}_{3} \mathrm{~N}_{2}$ occurs and it is believed that this is the reason causing the polarity inversion [36]. Theoretical calculations [44] and experimental observations [45-48] revealed that in PIDs, a single Mg layer is inserted between two polarity inverted GaN layers. This configuration constitutes the upper [0001] boundary of the PIDs .

4. Compensating defects

Several defects related to $\mathrm{Mg}$ doping are present in MOCVD grown GaN. These defects like the $\mathrm{Mg}$ interstitial $\left(\mathrm{Mg}_{\mathrm{i}}\right)$, the complex of $\mathrm{Mg}$ substitutional to Ga combined with nitrogen vacancy $\left(\mathrm{Mg}_{\mathrm{Ga}}-\mathrm{V}_{\mathrm{N}}\right)$ and the single $\mathrm{V}_{\mathrm{N}}$ are compensating the effect of the acceptor dopant in $\mathrm{GaN}$ [49]. Specifically, $\mathrm{Mg}_{\mathrm{i}}$ acts like a double donor as the $\mathrm{Mg}_{\mathrm{Ga}}-\mathrm{V}_{\mathrm{N}}$ while, $\mathrm{V}_{\mathrm{N}}$ is the most stable single donor defect in $p-\mathrm{GaN}$ [33]. When the
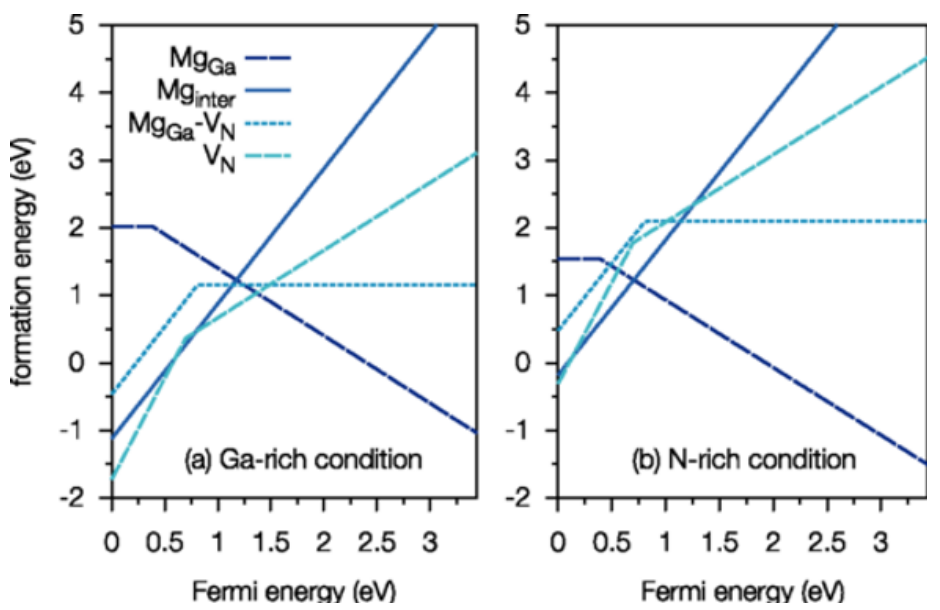

Figure 1.7: Defects formation energies in relation to the Fermi level in Mgdoped $\mathrm{GaN}$ grown under a) $\mathrm{Ga}$ - or $b$ ) $\mathrm{N}$ - rich conditions. The formation energies were calculated using hybrid-DFT. Reprinted with permission from \{https://doi.org/10.1103/PhysRevB.93.165207\}. Copyright \{2022\} American Physical Society. Ref.[33]. 
p-type doping increases, the Fermi energy drops to lower level. As shown in Fig. 1.7 [33], Miceli et al. have shown using hybrid-DFT that lowering of the Fermi level leads to an increase of the formation energy for the $\mathrm{Mg}_{\mathrm{Ga}}$ acceptor while the formation energies for the compensating defects is decreased. Therefore, increasing the $\mathrm{Mg}$ doping concentration makes the formation of compensating defects easier than the formation of $\mathrm{Mg}$ substitutional to $\mathrm{Ga}$ and consequently reducing the free hole concentration in GaN. Additionally, further decrease of the free hole concentration could be induced by the formation of $\mathrm{Mg}_{3} \mathrm{~N}_{2}$ and segregated $\mathrm{Mg}$ atoms in clusters, that effectively reduce the available $\mathrm{Mg}$ atoms for incorporation at the Ga-site.

5. Unintentional dopants and post-growth annealing

As already mentioned at the beginning of this section, unintentional doping is always present in MOCVD processes and it is related to the high growth pressure and to residual impurities in the chamber, as well as in the precursors and in the carrier gases. Contributing elements to the unintentional impurities in the growth of $p$ type $\mathrm{GaN}$ are: the $\mathrm{Si}, \mathrm{O}, \mathrm{C}$ and $\mathrm{H}$. Although $\mathrm{Si}$ and $\mathrm{O}$ form shallow donors, their concentration can be limited in our hot-wall MOCVD process to $\sim 3-5 \times 10^{15} \mathrm{~cm}^{-3}$, which is close to the SIMS detection limits for these elements $\left(\sim 2 \times 10^{15} \mathrm{~cm}^{-3}\right)$. The amphoteric role of $\mathrm{C}$ was also discussed at the beginning of this section while the compensating effects of $\mathrm{C}$ in $p$-GaN are discussed in detail in the literature $[45,50]$ Similarly to $\mathrm{Si}$ and $\mathrm{O}, \mathrm{C}$ can be controlled by the growth process parameters down to $\sim 5 \times 10^{15} \mathrm{~cm}^{-3}$.

The last impurity element to be discussed, $\mathrm{H}$, is abundant in the MOCVD process as a result of the carrier gas mixture $\left(\mathrm{H}_{2}\right.$ and $\left.\mathrm{N}_{2}\right)$ and the cracking of $\mathrm{NH}_{3} . \mathrm{H}$ passivates the $\mathrm{Mg}$ acceptor by formation of $\mathrm{Mg}-\mathrm{H}$ complexes resulting in equal concentrations of $\mathrm{Mg}$ and $\mathrm{H}$ in the $\mathrm{GaN}$ lattice. This mechanism is valid up to a $\mathrm{Mg}$ doping limit of $\sim 3 \times 10^{19} \mathrm{~cm}^{-3}$ when the $\mathrm{H}$ stops to incorporate in the same rate as $\mathrm{Mg}$, denoting that the $\mathrm{Mg}-\mathrm{H}$ complex formation ceases. Consequently, the $\mathrm{Mg}$ atoms are no longer incorporated in the Ga substitutional site rather than forming other complexes or interstitials as mentioned before. The interstitial hydrogen $\left(\mathrm{H}_{\mathrm{i}}\right)$ in $p$-GaN behaves as a donor $\left(\mathrm{H}^{+}\right)$thus, compensating the acceptor role of $\mathrm{Mg}_{\mathrm{Ga}}$.

Nevertheless, first principle calculations by Van de Walle et al. show that H-rich environment during Mg-doped GaN growth would be beneficial in suppressing the formation of native defects $\left(\mathrm{V}_{\mathrm{N}}\right)$ and at the same time enhance the $\mathrm{Mg}$ incorporation. The formation energy of $\mathrm{H}^{+}$is similar but lower than the formation energy of $\mathrm{V}_{\mathrm{N}}^{+}$. Consequently, the $\mathrm{E}_{\mathrm{F}}$ at equilibrium, which is located near the point that the formation energy of $\mathrm{Mg}_{\mathrm{Ga}}^{-}$equals the formation energy of $\mathrm{V}_{\mathrm{N}}^{+}$, is shifted at a higher energy level, where the formation energy of $\mathrm{Mg}_{\mathrm{Ga}}^{-}$is lower. As a result, $\mathrm{Mg}$ 
incorporation is enhanced. An investigation based on this concept was done and is presented in Paper II of this thesis.

Since the incorporated $\mathrm{Mg}$ is almost entirely passivated by $\mathrm{H}$, a post-growth thermal annealing treatment is required to release the $\mathrm{H}$ and render electrically active $\mathrm{Mg}$ acceptors. Post-growth rapid or regular thermal annealing at temperatures between $750-900^{\circ} \mathrm{C}$ under $\mathrm{N}_{2}$ atmosphere is usually performed in order to activate the $\mathrm{Mg}$ in $p$-GaN. There are studies suggesting that different annealing temperatures are suitable for different $\mathrm{Mg}$ concentrations in $p-\mathrm{GaN}$ [51]. According to Liu et al., the process of $\mathrm{Mg}$ related donors transferring to non-radiative recombination centers is dominating over $\mathrm{Mg}$ acceptor activation in heavily doped $p-\mathrm{GaN}$ as the annealing temperature increases. In the current study, we annealed the samples at $900^{\circ} \mathrm{C}$ under $\mathrm{N}_{2}$ atmosphere for 10 minutes to activate the $\mathrm{Mg}$ acceptors.

6. Memory effect

Another obstacle in $\mathrm{Mg}$ doping process in $\mathrm{GaN}$ is the delayed incorporation and the persistence upon $\mathrm{Mg}$ incorporation stopping, the so-called memory effect. The $\mathrm{Mg}$ precursor molecules have the tendency to stick on the gas pipes and the growth chamber walls which results in a delayed initiation of $\mathrm{Mg}$ incorporation in $\mathrm{GaN}$, after introducing the $\mathrm{Mg}$ precursor in the chamber. Additionally, the $\mathrm{Mg}$ atoms are accumulating on the GaN surface [52] during GaN:Mg growth and therefore, $\mathrm{Mg}$ is incorporated into subsequent layers. Obviously, it is extremely difficult to acquire a step-like profile of $\mathrm{Mg}$ doping in $\mathrm{GaN}$ although, a possible way for sharpening the profile onset is by high $\mathrm{Cp}_{2} \mathrm{Mg}$ pre-flow upon start of $\mathrm{GaN}: \mathrm{Mg}$ growth.

\subsection{MOCVD growth of Mg-doped GaN and AlGaN/GaN HEMTs}

MOCVD is the most commonly used growth technique for epitaxially grown electronic and opto-electronic devices. Epitaxial growth of III-nitrides with outstanding properties have been demonstrated by MOCVD while, the development of this technique over the last three decades led to low impurity levels in as-grown materials and effective ways of creating sharp interfaces in device structures. These benefits in combination with the high growth rates enabled by MOCVD while maintaining the material quality and purity have put MOCVD in leading position for epitaxial growth of semiconductors compared to other growth techniques. 


\subsubsection{MOCVD process fundamentals}

During the MOCVD process, precursors in vapor phase undergo chemical reactions leading to solid material deposition on the substrate. metal-organic (MO) precursors (group-III element sources) and ammonia $\left(\mathrm{NH}_{3}\right)$ (group-V element source) are used for the growth of III-nitride semiconductor materials. External energy in the form of heat is necessary for the precursors pyrolysis and the release of atomic species for reaction. As gaseous precursors are used for the MOCVD, the process relies on surface chemical reactions. MOCVD is a process with high complexity because, it incorporates gas flow dynamics, chemistry and thermodynamics, with numerous parameters being involved in the process [53]. Nevertheless, a simple description of MOCVD could be given by the main general steps, as depicted in Fig. 1.8:

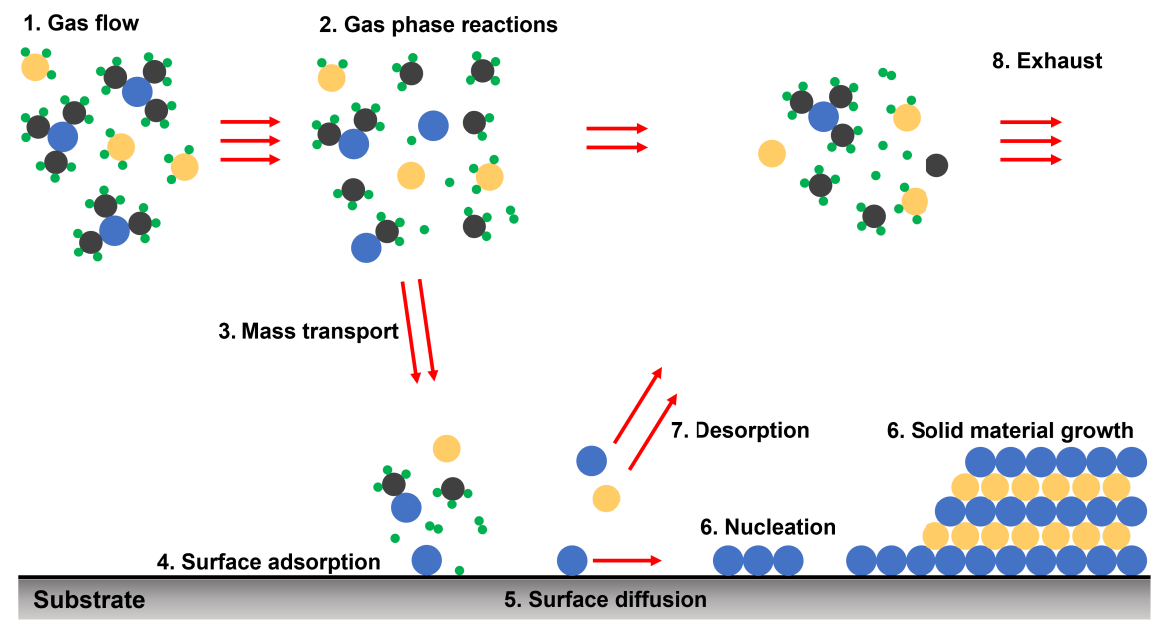

Figure 1.8: Schematic view of the physical processes involved in MOCVD growth.

1. Precursors in gas phase are transported to the growth zone.

2. Gas-phase reactions of the precursors at the hot zone. Formation of reactive intermediates and by-products.

3. Mass transport (diffusion) of precursors and reactants through the boundary layer to the substrate surface.

4. Surface reactions and adsorption of reactants on the surface . 
5. Surface diffusion of atoms to the growth sites.

6. Nucleation and surface reactions resulting in growth of solid material.

7. Surface desorption and mass transport of by-products away from the growth zone.

8. Non-reacted precursors and growth by-products are pumped out to the exhaust.

\subsubsection{Gas flow and growth pressure}

The MOCVD process is mainly controlled by the mass transport from gas phase to the substrate, thus, it follows the gas flow dynamics. For achieving high uniformity of the growth rate and of the solid material composition over the whole substrate, the growth process should be performed under laminar gas flow conditions. In such case, the gases flow in parallel layer above the substrate surface with no formation of turbulent gas flow patterns. The flow regime in a viscous system can be described by the Reynolds number $(R e)$ Eq. 1.7,

$$
R e=\frac{\text { Inertia forces }}{\text { viscous forces }}=\frac{u L}{v}=\frac{\rho u L}{\mu}
$$

where, $u$ is the mean velocity of the fluid, $L$ is the distance travelled by the fluid (linear dimension), $v$ is the kinematic viscosity of the fluid, $\rho$ is the fluid density and $\mu$ is the dynamic viscosity. For $R e<2000$ the gas flow is laminar while for $R e>2500$ the turbulent flow is initiated and inter-mixing of the gas layers happens. Maintaining the laminar flow for the growth process depends a lot on the design (geometrical aspects) of the MOCVD chamber.

The mass transfer from the gas phase to the substrate surface happens via diffusion of the molecules and radicals through the so-called boundary layer. The precursor molecules concentration is gradually decreasing closer to the substrate where most of the reactants are consumed to form the layer. Thus, the precursor concentration gradient within the boundary layer leads to mass diffusion from the higher concentration in the gas flow to almost zero concentration near the substrate surface resulting in reactant molecules flux towards the substrate. When a gas flows in a parallel plane over a flat surface, the gas velocity decrease from its max value $u_{0}$ to zero as we move from the free gas stream to the substrate surface (or the chamber walls if we assume an empty cylindrical chamber). Frictional forces acting on the gas molecules are responsible for this effect. Boundary layer with thickness $\delta(x)$, Eq. 1.8, is then defined as the gas layer for which the gas velocity is lower than $u_{0}$ as depicted in Fig. 1.9.

$$
\delta(x) \propto \frac{x}{\sqrt{\operatorname{Re}}}=x \sqrt{\frac{\mu}{\rho u_{0} L}}
$$




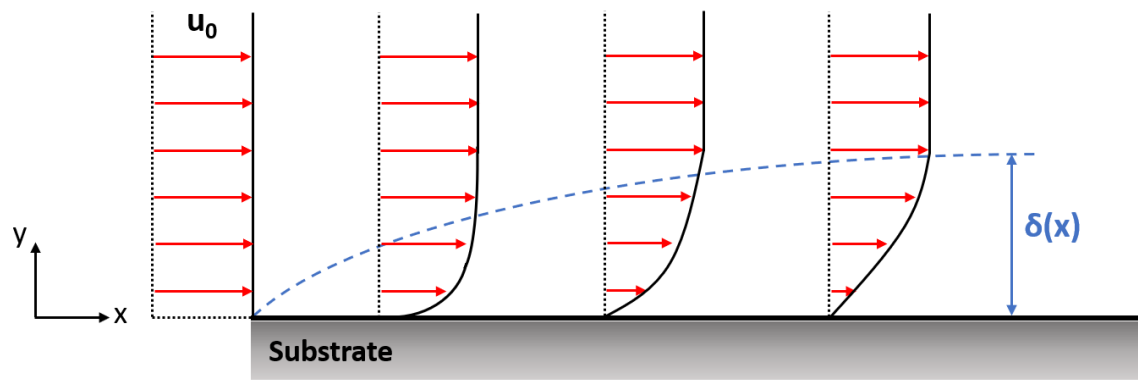

Figure 1.9: Gas velocity profile over a flat surface and formation of the boundary layer with thickness $\delta(x)$.

Since the reactants must diffuse through the boundary layer to reach the substrate, the boundary layer thickness is a very important factor determining the growth rate. In a thin boundary layer, the reactants can easily diffuse to the substrate but their concentration in the boundary layer is quickly depleted at the downstream end of the deposition region [54]. For a homogeneous layer growth, a relatively thick boundary layer is needed. Therefore, controlling the thickness of the boundary layer can be achieved by adjusting the gas velocity, which practically means to adjust either the total gas flow or the pressure. In our process we mainly adjust pressure as the process parameter.

The MOCVD of III-nitrides is favored by a relatively low growth pressure (50100 mbar) in several ways. Besides adjusting the boundary layer thickness as already mentioned, low pressure reduces the possibility of turbulent flow patterns formation, thus, minimizing the molecules interaction in the gas phase, which in turn means less parasitic reactions. This effect becomes more prominent in the III-nitrides growth process for semiconductor quality material where the growth temperature is high and the prereactions at the gas phase occur with higher rates. Nevertheless, the process pressure should not be very low either, because it enhances the unintentional incorporation of impurities (mainly C) [55] which is detrimental for semiconductor quality materials.

\subsubsection{Growth temperature}

As mentioned earlier, an external energy source is needed to activate the reaction process for the precursor molecules. Energy in the form of heat is supplied to the system by increasing the temperature. The growth rate in this case is affected by the substrate temperature and can be divided in three different regimes relating the growth temperature with the major factor limiting the growth rate: $i$ )low temperature regime where the surface 
kinetics become important and the growth rate is reaction rate limited, ii) medium-tohigh temperature regime where the growth rate is mass transport limited and iii) high temperature regime where the thermodynamics become important and the growth rate is limited by parasitic reactions and decomposition [56].

i) Low temperature regime

The growth rate in the low temperature regime increases exponentially with temperature, because it is limited by the reaction kinetics. In this regime, the rates of several thermally activated processes, like the gas phase reactions, diffusion of adatoms at the surface and nucleation, are greatly affected by small temperature variations. In this case, the slowest reaction is limiting the growth rate because it has the higher activation energy. Consequently, the growth rate becomes very sensitive to small changes in temperature leading to unstable and non-reproducible material growth. It should also be noted that unintentional impurities incorporation is more pronounced when the growth of III-nitrides is performed in this regime.

ii) Medium-to-high temperature regime

In the medium temperature regime the growth rate is not affected by the temperature but only by the mass transport to the substrate surface, in other words to the diffusion rate of reactants through the boundary layer. Since the growth rate is proportional to the reactants flux J (Eq. 1.9) in the mass transport limited regime, we can alter the precursor concentration or the boundary layer thickness in order to change the growth rate.

$$
J=\frac{D\left(P_{g}^{0}-P_{s}\right)}{R T \delta} \propto \text { growth rate }
$$

where $J$ is the the flux of reactants, $P_{g}$ and $P_{s}$ are the partial pressures of the III-group element in the gas phase and at the substrate surface respectively, $\mathrm{D}$ is the diffusion coefficient of the group-III element in the carrier gas and $R$ is the gas constant. Except from the increase of precursor concentration in the gas phase, the flux $J$ can be increased by reducing the boundary layer thickness or increase the diffusivity $D$. The diffusivity can be increased by reducing the process operating pressure. Reduction of the pressure would further lead to decrease of the boundary layer thickness which is crucial for the precursor flux to the surface and consequently for the layer uniformity. Therefore, in our growth process we opt for a pressure range that allows for uniform and low impurity level depositions and we adjust the growth rate by the amount of precursor material introduced in the gas phase. 


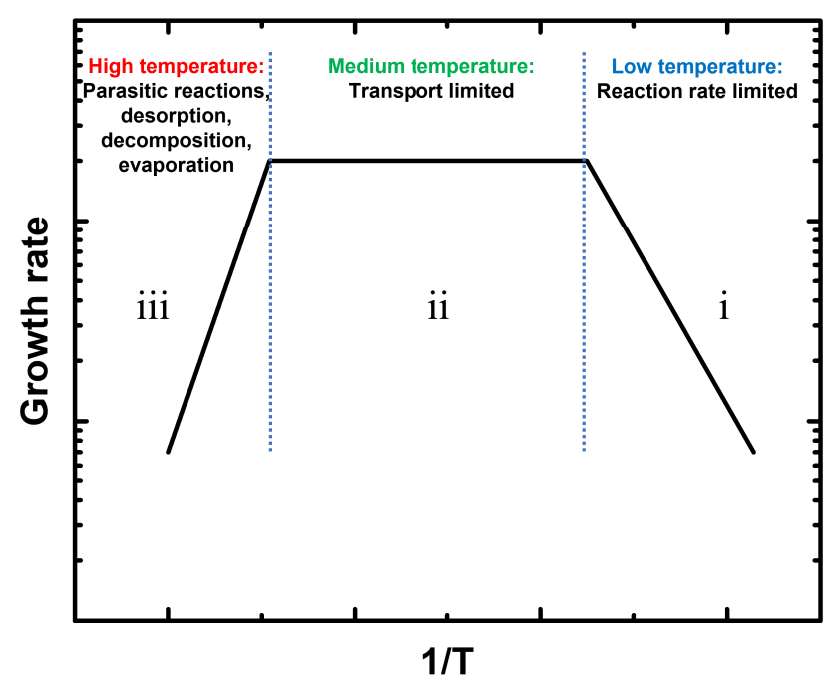

Figure 1.10: Growth rate regimes related to growth temperature

iii) High temperature regime

In the high temperature growth regime, the growth rate is significantly reduced due to thermodynamic limitations. The driving force of growth, the supersaturation is decreased leading to increased desorption rate from the substrate surface. Several other processes related to the high temperature such as parasitic reactions in the gas phase (reduction of available reactants) and material decomposition due to increased vapor pressure of the solid material, lead to decrease of the growth rate.

\subsubsection{Supersaturation}

The MOCVD growth process relies on the minimization of the Gibbs free energy $(G)$ in order to restore the vapor-solid system in equilibrium state. Eventually, the minimization of $G$ becomes the driving force for growth [57].

$$
\Delta G=-R T \ln (1+\sigma)
$$

Initially, the system consisting of molecules in the gas and solid phase is brought to non-equilibrium conditions when temperature and pressure are changed. Under these 
conditions, the difference in chemical potential $\Delta \mu$, between the vapor and solid phases i.e. supersaturation $(\sigma)$ is created, and must be consumed towards solid material growth. Increasing the process temperature, drives the system further away from the equilibrium condition and consequently increases the driving force for growth.

$$
\sigma=\frac{P_{G a}^{0}-P_{G a}}{P_{G a}}
$$

Eq. 1.11 defines the $\mathrm{Ga}$ supersaturation, where $\mathrm{P}_{\mathrm{Ga}}^{0}$ is the input partial pressure of $\mathrm{Ga}$ species and $\mathrm{P}_{\mathrm{Ga}}$ is the equilibrium vapor pressure of $\mathrm{Ga}$ above $\mathrm{GaN}$ at the growth temperature.

The Ga supersaturation in the GaN MOCVD for this thesis is calculated following Mita et al. [57]. Ga supersaturation is an easy-to-handle parameter containing information about the growth parameters in practice, and can simplify the understanding on the highly complex process of MOCVD growth. Nevertheless, other parameters should be always taken into account (e.g. the dopant/Ga precursor molecules ratio) in order to describe and/or predict the MOCVD process outcome. The C levels for instance, in Mg-doped GaN show linear increase with supersaturation. This effect can be related to either the growth temperature variation, or the amount of MO precursors in gas phase.

\subsubsection{Hot-wall MOCVD concept}

Hot-wall MOCVD is a unique modification of MOCVD reactor. State-of-the-art $\mathrm{SiC}[58,59]$ and group-III nitride materials [31, 60, 61] were demonstrated by hot-wall MOCVD in recent years. Hot-wall MOCVD is capable of delivering material at industry-relevant growth rates while maintaining the superior material purity and structural quality. A simplified schematic of a horizontal gas flow hot-wall MOCVD system is shown n Fig. 1.11. In such system, the carrier gases and the precursors in gas phase are delivered to the system and transported to the hot zone where the substrate is located. In the high temperature environment, the precursors react to form epitaxial layers on the heated substrate. The by-products and exhaust gases are pumped out of the chamber and pass through a filtering system (scrubber) to be neutralized before releasing environmental friendly gases to the atmosphere. The pressure during growth is controlled and maintained by the pumping system.

A mixture of nitrogen $\left(\mathrm{N}_{2}\right)$ and $\mathrm{H}_{2}$ is used as carrier gas for the $\mathrm{MO}$ precursor molecules i.e. TMGa, trimethylaluminum (TMAl) and $\mathrm{Cp}_{2} \mathrm{Mg}$ in the reactor. $\mathrm{N}_{2}$ and $\mathrm{H}_{2}$ pass through purifiers before use. The $\mathrm{MO}$ source materials, either liquid or solid, are stored in cylinders 


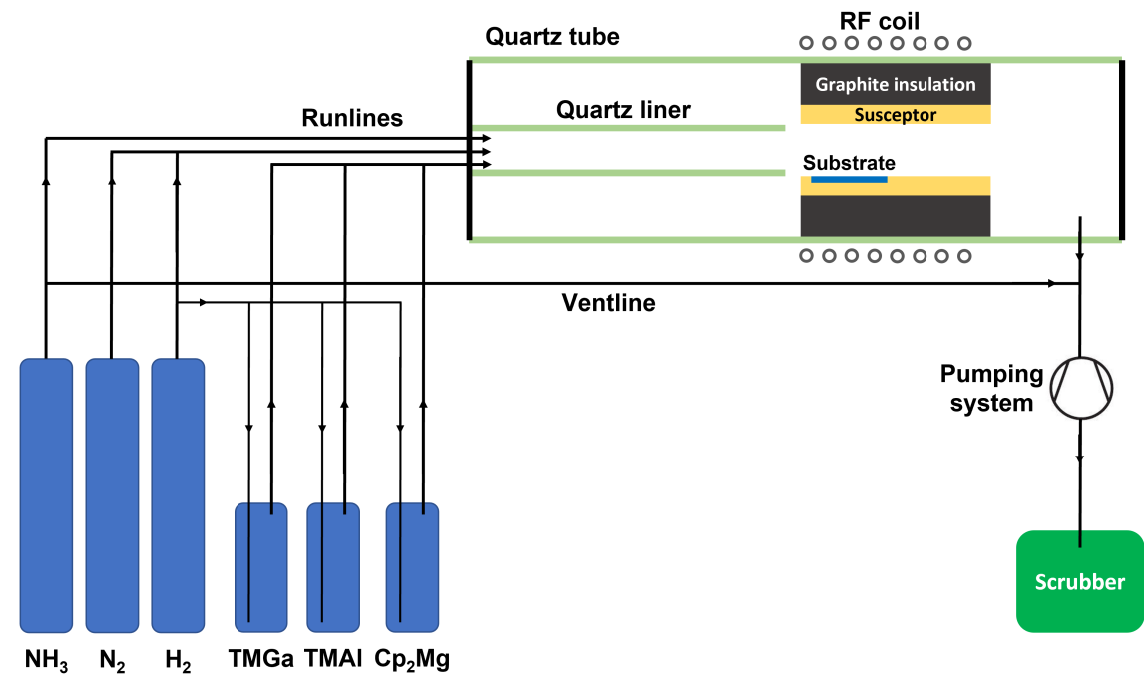

Figure 1.11: Simplified schematic diagram of the hot-wall MOCVD

(bubblers) which are immersed in temperature controlling baths. $\mathrm{H}_{2}$ gas is flown through the bubblers and gets enriched with the volatile MO material. Since this process is highly dependent on the MO vapor pressure in the bubbler and the amount of $\mathrm{H}_{2}$ passing through it, the bubbler temperature must be very stable and the $\mathrm{H}_{2}$ flow must be accurately controlled, respectively. For the latter, every gas flow in the MOCVD is controlled by mass flow controller (MFC) and there is the possibility to switch each gas to the reactor or to the vent by separate control valves. The gas mixture of $\mathrm{H}_{2}$ and MOs is delivered to the reactor via the $\mathrm{MO}$ runline. The atomic $\mathrm{N}$ precursor, $\mathrm{NH}_{3}$, is purified and then delivered through a separate line, the hydride runline, to avoid intermixing of precursors prior to the growth zone that could activate pre-reactions. As the gases enter the reactor, they flow through a quartz liner that enables the formation of laminar flow and transports the reactants to the growth zone.

The main difference between a hot wall and a cold wall MOCVD system is the way that the substrate is heated. Fig. 1.12 shows a schematic representation of the hot zone of the hot-wall MOCVD reactor used in this work (VP508GFR, Aixtron). The system has a capacity of $1 \times 4^{\prime \prime}$ or $1 \times 3^{\prime \prime}$ or $3 \times 2^{\prime \prime}$ wafers depending on the satellite used.

Heat is provided by current induction in the graphite susceptor parts which form a pocket environment around the substrate. The induction currents are formed by a coil 


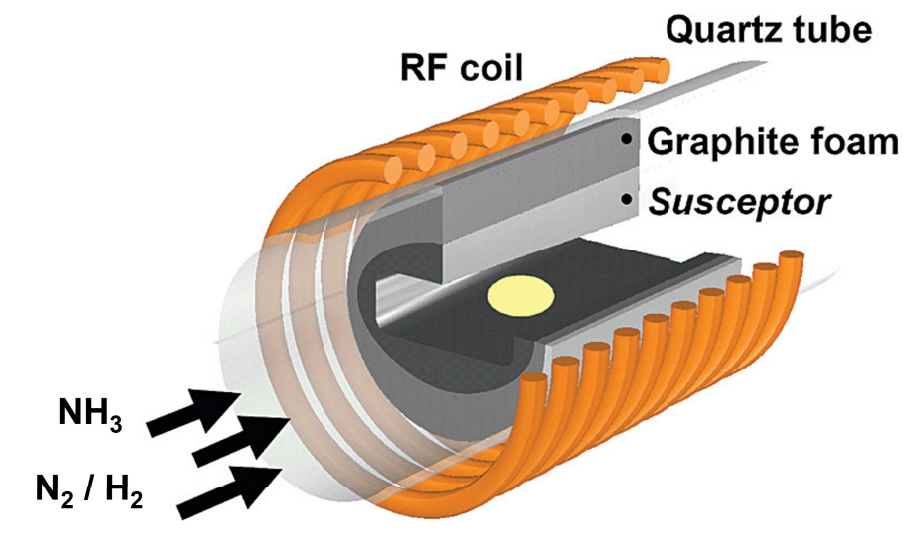

TMGa / TMAI / $\mathrm{Cp}_{2} \mathrm{Mg}$

Figure 1.12: Schematic representation of the hot zone in the hot wall MOCVD reactor used in this thesis. The precursors and carrier gases pass through the hot zone above the substrate (disk at the lower surface of susceptor). Reprinted with permission from \{Crystal Growth \& Design 2009, 9, 2, 880-884\}. Copyright \{2022\} American Chemical Society. Ref.[6o]

wrapped around the quartz tube chamber when is operated at RF. The system can easily reach temperatures over $1400^{\circ} \mathrm{C}$. All the graphite susceptor parts are coated with tantalum carbide $(\mathrm{TaC})$ which protects the graphite against corrosion under the harsh environment of $\mathrm{NH}_{3}$ and its decomposition products at high temperature [62].

The substrate sits on the satellite which is placed on the satellite carrier. The satellite rotates during the growth using a gas-foil-rotation (GFR) system. The substrate rotation is very important for achieving uniform layers because it compensates for the reactants' depletion in the linear growth profile. Rotation is also important for maintaining the uniform temperature distribution on the satellite and consequently on the substrate.

The whole susceptor construction is surrounded by graphite foam which is used as thermal insulator to maintain the susceptor temperature and protect the quartz tube from melting. The susceptor temperature is continuously monitored and measured by a pyrometer. After the reaction in the hot zone, by-products and exhaust gases are pumped out of the chamber and pass through the scrubber system for neutralization before releasing to the atmosphere. A mechanical dry pump is used for regulating the process pressure which is in the range of $50-100$ mbar. Background gases introduce impurities in the material which is detrimental for the electrical properties of semiconductors. Therefore, 
an additional turbo-molecular pump is used prior to growth process for reducing the background pressure in the chamber to low $10^{-5} \mathrm{mbar}$.

In general, the hot-wall MOCVD concept used here provides several advantages compared to the commonly used cold wall MOCVD reactors where only the substrate is resistively heated. In the hot-wall reactor configuration the whole susceptor is heated creating a hot zone with much higher uniformity which results in highly uniform epitaxial layers [61, 63]. In addition, several examples of III-nitride materials with exceptional quality, relevant for application in high-power high-frequency electronics $[6,31,64]$ as well as in quantum technology [65], were developed in this hot-wall MOCVD reactor.

\subsubsection{Epitaxy of Mg-doped GaN}

The process of acquiring $p$-type GaN consists of several steps with important consideration to be taken into account. These considerations are related to the choice of substrate, the substrate pre-treatment, the epilayers growth conditions and finally, the post-growth annealing conditions.

\section{Choice of substrate}

Power and RF devices require good thermal dissipation from the active layers to the substrate thus, substrates with high thermal conductivity are needed for epitaxial growth of such structures. Additionally, the substrate should have similar in-plane lattice constant with the epilayers. The lattice mismatch between substrate and epilayer causes strain which is released through formation of defects, e. g., dislocations in the epilayer which are detrimental for device performance, as they serve as current leakage paths. In order to accommodate the lattice mismatch, several strain engineered layers should be grown before the active layers resulting in more interfaces, which deteriorate the thermal conduction efficiency to the substrate. For these reasons, the choice of substrate is crucial as it is the basis of the whole growth process. GaN epitaxy was initially developed on foreign substrates since bulk GaN substrates were not available. The most commonly used substrates for GaN epitaxy are listed on Table 1.2 along with their basic properties. As can be seen from the table, sapphire and Si have very large lattice mismatch with GaN though they are the cheapest choice. On the other hand, bulk GaN has very high cost although it renders the best quality epilayers in terms of dislocation density, because of the homo-epitaxial growth. Additionally, GaN has high thermal conductivity, a crucial factor when designing devices for high power applications. The best alternative to combine high thermal conductivity and low lattice mismatch with $\mathrm{GaN}$ is SiC. Semi-insulating $\mathrm{SiC}$ substrates are available in large wafer size (8-inch) and although 
Table 1.2: Material properties of $\mathrm{Si}$, Sapphire, ${ }_{4} \mathrm{H}-\mathrm{SiC}$, and $\mathrm{GaN}$.

\begin{tabular}{|c|c|c|c|c|}
\hline & $\mathrm{Si}^{a, g}$ & Sapphire ${ }^{e, g}$ & $4 \mathrm{H}-\mathrm{SiC}^{a, f}$ & $\mathrm{GaN}^{a, b, c, d}$ \\
\hline Bandgap $(\mathrm{eV})$ & 1.12 & $8.10-8.60$ & 3.26 & 3.4 \\
\hline \multicolumn{5}{|l|}{ Lattice constants @300 K $(\AA)$} \\
\hline$a_{0}$ & 5.4310 & 4.7650 & 3.0798 & 3.1893 \\
\hline$c_{0}$ & & 12.9820 & 10.0820 & 5.1852 \\
\hline In-plane lattice mismatch (\%) & 20.0 & -13.7 & -3.5 & 0 \\
\hline \multicolumn{5}{|l|}{ Thermal expansion coefficients } \\
\hline$a_{\mathrm{a}}\left(10^{-6} \mathrm{~K}^{-1}\right)$ & 2.6 & 5.0 & 3.30 & 5.59 \\
\hline$a_{\mathrm{c}}\left(10^{-6} \mathrm{~K}^{-1}\right)$ & & 9.03 & 3.16 & 3.17 \\
\hline Thermal conductivity $\left(\mathrm{Wcm}^{-1} \mathrm{~K}^{-1}\right)$ & 1.3 & 0.25 & 4.9 & 2.3 \\
\hline $\begin{array}{l}\text { Dislocation density of epi-GaN } \\
\left(\mathrm{cm}^{-2}\right)\end{array}$ & low $10^{8}$ & low $10^{8}$ & low $10^{8}$ & $10^{4}-10^{6}$ \\
\hline Cost & cheap & cheap & expensive & very expensive \\
\hline
\end{tabular}

their cost is much higher than sapphire and $\mathrm{Si}$, they are still cheaper than bulk GaN. $4 \mathrm{H}-\mathrm{SiC}$ substrates were used for the development of $p$-GaN growth process in this thesis. $n$-type $4 \mathrm{H} \mathrm{SiC}$ from Cree Inc. is used for GaN growth optimization and SI- $4 \mathrm{H}$ $\mathrm{SiC}$ from Norstel AB and SICC for Mg-doped GaN.

2. Substrate pre-treatment

A high-temperature pre-treatment of the $\mathrm{SiC}$ substrates under $\mathrm{H}_{2}$ environment is required to form a step-like surface morphology which promotes the step-flow growth of the III-nitride epilayers. After dicing, the chemical mechanical polished (CMP) $\mathrm{SiC}$ substrates are chemically cleaned for removing residual organic contaminants and particles. Before growth start of the epilayers, the high temperature pre-treatment is performed in order to remove any contaminants and the oxide formed on the $\mathrm{SiC}$ surface. During this process, the $\mathrm{Si}$ atoms are evaporated due to the high temperature and the $\mathrm{C}$ atoms react with the $\mathrm{H}_{2}$ to form hydrocarbon gases, etching in this way the $\mathrm{SiC}$ surface [70]. If the evaporation rate of $\mathrm{Si}$ atoms equals the C-to-hydrocarbon conversion rate, then a step-like surface morphology will be formed [71]. The step-flow etching is required to depress the defect formation on $\mathrm{SiC}$ surface upon preparation for epilayer growth [61].

3. AlN nucleation layer

A nucleation layer between $\mathrm{SiC}$ and $\mathrm{GaN}$ is required to accommodate the large lattice mismatch $(\sim 3.5 \%)$ and to provide a 2-dimensional surface for GaN growth. Since $\mathrm{AlN}$ has $\sim 1 \%$ lattice mismatch with $\mathrm{SiC}$, it serves as a useful transition layer 
reducing the mismatch hence, preparing the surface for GaN growth [72]. Direct growth of $\mathrm{GaN}$ on $\mathrm{SiC}$ results in non-continuous layers because of surface wetting problems $[73,74]$. AlN nucleation layer was grown on all the structures included in the thesis at a temperature of $1250^{\circ} \mathrm{C}$ and V/III ratio $\sim 1260$ with a growth rate of $0.33-0.36 \mu \mathrm{m} / \mathrm{h}$. A thickness of $\sim 60 \mathrm{~nm}$ was enough for providing a good template for subsequent $\mathrm{GaN}$ growth.

4. GaN buffer layer

The AlN nucleation layer has high-density threading dislocations, which originate from the strain relaxation in this layer when its thickness goes beyond the critical thickness. The dislocations propagate in the subsequent GaN epilayer and their density reduces with increasing GaN thickness as moving away from the AlN/GaN interface. The density of edge- $(a)$ - type or mixed- $(a+c)$ - type threading dislocations (TDs) is one order of magnitude higher than the screw- (c)- type TDs for GaN on $\mathrm{SiC}$. In our study for $p$-type GaN growth, $1 \mu \mathrm{m}$ thick UID-GaN served as a buffer layer for the Mg-doped layer at the optimized structures. The edge- and screw- type dislocation densities in these structures were $\sim 5 \times 10^{8} \mathrm{~cm}^{-2}$ and $\sim 2 \times 10^{7} \mathrm{~cm}^{-2}$ respectively. The buffer layer should be highly resistive to avoid current leakages. For this reason, minimizing the unintentional doping impurities is crucial and can be done by tuning of the growth conditions.

\section{GaN:Mg}

The $\mathrm{Mg}$ doping in $\mathrm{GaN}$ was thoroughly discussed in section 1.2.5. GaN:Mg layers with $\mathrm{Mg}$ concentration in the range of $2.5 \times 10^{18} \mathrm{~cm}^{-3}-1.1 \times 10^{20} \mathrm{~cm}^{-3}$ were grown for investigation of the $\mathrm{Mg}$ incorporation and the effect of background impurities on the structural and electrical properties of these layers (Paper I). The $p$-GaN was directly grown on the AlN nucleation layer, except for the optimized structure that was grown on $1 \mu \mathrm{m}$ thick GaN buffer layer (see previous paragraph). Since $500 \mathrm{~nm}$ thick layers have higher dislocation density when grown directly on the nucleation layer instead of growing on a buffer $\mathrm{GaN}$, the necessity of a GaN buffer layer becomes obvious from the improvement of electrical characteristics when deploying the GaN buffer (Fig. 1.13).

6. Post-growth annealing

A post-growth annealing step is necessary for hydrogen dissociation from the $\mathrm{Mg}-\mathrm{H}$ complexes and out-diffusion in order to activate the $\mathrm{Mg}_{\mathrm{Ga}}$ acceptors. This process can be performed either by rapid thermal annealing (RTA) or regular annealing under $\mathrm{N}_{2}$ or $\mathrm{O}_{2}$ [75-77] atmosphere. Commonly, $\mathrm{N}_{2}$ atmosphere is used for suppressing $\mathrm{N}$ dissociation from $\mathrm{GaN}$ at high temperatures $\left(750-900^{\circ} \mathrm{C}\right)$. In this study, we used 


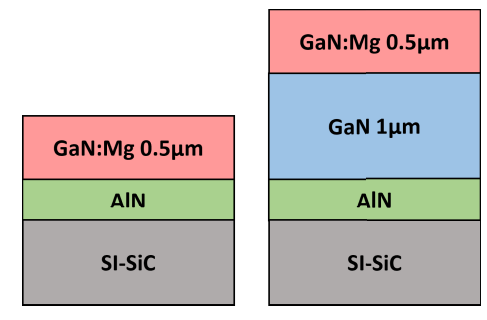

Figure 1.13: Schematic representation of the Mg-doped GaN samples grown on semiinsulating $\mathrm{SiC}$ substrate either with- or without $\mathrm{GaN}$ buffer layer

both a conventional annealing method with annealing parameters $900^{\circ} \mathrm{C}$ under $\mathrm{N}_{2}$ atmosphere for 10 minutes, and RTA at $900^{\circ} \mathrm{C}$ in order to activate the $\mathrm{Mg}$ acceptors. It should be mentioned that we observed $p$-type conductivity in the as-grown samples without the need to anneal which is a unique advantage of GaN:Mg growth by hot-wall MOCVD and the specific growth parameters used (Paper I and Paper II).

\subsubsection{Epitaxy of AlGaN/GaN HEMTs}

The growth process of HEMT structures consists of several different steps, each of them requires optimization in order to acquire the desired properties. The growth process of a basic HEMT structure is described below. In this we work, the presented results include only investigation of the most basic AlGaN/GaN HEMT structure. The properties of this type of structures sre discussed in detail in section 1.2.2 while a schematic of the structure is shown in Fig. 1.14.

The choice of substrate, the substrate pre-treatment and the AlN layer growth steps are the same as described in section 1.3.3 for the $p$-GaN growth process thus, they will only be referenced here.

1. Choice of substrate $\rightarrow$ same as in section $1.3 \cdot 3$

2. Substrate pre-treatment $\rightarrow$ same as in section 1.3 .3

3. AlN nucleation layer $\rightarrow$ same as in section $1.3 \cdot 3$

4. GaN buffer layer

The GaN buffer layer in a HEMT structure has several roles as already discussed for the GaN:Mg structures. Specifically for the HEMTs, the GaN buffer layer is required to provide atomically flat surface at the channel region where the 2DEG is formed. Moreover, it reduces the threading dislocations density in order to minimize 
the current leakage paths (note that the threading dislocations intersect the 2DEG) while it also prevents the parallel conduction in the structure. For the latter, GaN buffer layers must be semi-insulating and this property can be achieved in various ways. As already mentioned, tuning of the growth conditions in MOCVD will render intrinsically semi-insulating GaN. Other ways to increase the resistivity of the GaN buffer layer is to intentionally dope it with acceptor impurities, most commonly $\mathrm{C}$ or Fe. C-doping may be realized by adjusting the growth conditions. As previously discussed, the residual $\mathrm{C}$ from the TMGa cracking can be incorporated in GaN if it is not transformed to $\mathrm{CH}_{4}$ gas. This process is highly related to the growth temperature and pressure as well as the amount of $\mathrm{NH}_{3}$. Of course, the available $\mathrm{C}$ amount for (un-)intentional incorporation in $\mathrm{GaN}$ is linearly related to the amount of TMGa when the rest of the growth parameters are not changed. $\mathrm{C}$ substitutional to nitrogen site in $\mathrm{GaN}\left(\mathrm{C}_{\mathrm{N}}\right)$, is a deep acceptor level $\sim 0.90 \mathrm{eV}$ above the valence band maximum [34, 78].

On the other hand, Fe is incorporated in $\mathrm{GaN}$ by introducing a metal-organic precursor, bis(cyclopentadienyl) iron $\left(\mathrm{Cp}_{2} \mathrm{Fe}\right)$ in the growth process. The Fe-doping is challenging in terms of sharp doping profile control, unlike C-doping, due to memory effects similarly to Mg-doping. The iron atom substitutes the Ga atoms in $\mathrm{GaN}\left(\mathrm{Fe}_{\mathrm{Ga}}\right)$ where it acts as atomic-like acceptor, with the $(0 /-)$ transition level being $3 \mathrm{eV}$ above the $\mathrm{GaN}$ valence band maximum. If $\mathrm{GaN}$ is mainly doped with $\mathrm{Fe}$, the $\mathrm{E}_{\mathrm{F}}$ is pinned at $0.5 \mathrm{eV}$ below the conduction band minimum [79]. It should be noted that high doping levels of such impurities, introduce traps that could either lead to electrons trapping from the 2DEG if they are in the proximity of the channel area, or cause current collapse in the HEMT device. For the samples presented in the current thesis, the GaN buffer layer was not doped (UID-GaN) while all the HEMT structures had a $1 \mu \mathrm{m}$ thick GaN buffer with $[\mathrm{C}] \sim 1 \times 10^{16} \mathrm{~cm}^{-3}$.

5. AlN interlayer (carrier exclusion layer)

The AlN exlusion layer is grown between the GaN buffer layer and the AlGaN barrier layer. The reason for introducing this layer is to create an abrupt band discontinuity between the channel and the barrier, and prevent the 2DEG wavefunction from penetrating the barrier layer. In this way, the alloy scattering is minimized as discussed in section 1.2.2 and results in higher mobility of the 2DEG. The thickness of the AlN interlayer is $\sim 1 \mathrm{~nm}$. In our structures, there was no AlN interlayer.

6. AlGaN barrier layer

The most important layer in a HEMT structure is the barrier layer because it defines the 2DEG properties which can be tuned by the Al-content and the thickness of 
the barrier. Higher Al-content in the AlGaN and larger barrier thickness lead to higher 2DEG density. A series of varied Al-content in AlGaN barrier (7-42\%) with $\sim 25-30 \mathrm{~nm}$ thickness is investigated in this thesis. Notably, all the barrier layers have to be grown pseudomorphically to $\mathrm{GaN}$ which can be verified by the XRD reciprocal space map (RSM)s. Increasing the Al-content in combination with the layer thickness could lead to plastic stain relaxation and cracking of the layer. Additionally, local elastic relaxation would lead to reduced strain field in the barrier layer and reduction of the 2DEG density. For these reasons, the AlGaN barrier growth should be treated with special care. The Al-content can be adjusted by the TMAl precursor over the total amount of metal-organic precursor gas phase ratio: $\frac{\text { TMAl }}{\text { TMGa+TMAl }}$. The mobility values in the investigated structures was in the range of $920-1345 \mathrm{~cm}^{2} \mathrm{~V}^{-1} \mathrm{~s}^{-1}$, while the 2DEG density scaled linearly from $4.8 \times 10^{12}$ to $1.5 \times 10^{13} \mathrm{~cm}^{-2}$ for Al-content of $7-42 \%$ respectively (Paper III).

\section{GaN cap layer}

The 2DEG transport properties are affected to a great extent by energy states in the near-surface area. As discussed before, the 2DEG density depends on the Al-content of the AlGaN barrier which directly affects the $P_{\mathrm{PE}}$. As the barrier layer is very thin (usually up to 30nm), introducing a thin cap layer has a double role for the HEMT structure: $a$ ) to protect the barrier layer from oxidation and $b$ ) to prevent the strain relaxation of the barrier layer over time or due to thermal stress during the HEMT operation [80, 81]. As a result, there is no deterioration of the AlGaN quality due to oxidation from the exposure to the environment. Additionally, the strain condition of the barrier layer is maintained, avoiding unwanted change in the $P_{\mathrm{PE}}$ which would be detrimental for the 2DEG density. The cap layer may also increase the biaxial tensile stain in the barrier layer leading to additional increase of the 2DEG density. At a device level, the cap layer improves the electrical characteristics such as the breakdown voltage [8o]. Furthermore, a cap layer on an AlGaN barrier could stabilize the surfacial composition of AlGaN by minimizing the peferential etching of $\mathrm{Ga}$ over $\mathrm{Al}$ atoms from this layer during the cooling down after growth process [82]. The most commonly used materials as HEMT cap layers are silicon nitride $\left(\mathrm{Si}_{3} \mathrm{~N}_{4}\right)$ and GaN with thicknesses ranging from $\sim 2-10 \mathrm{~nm}[83,84]$. The samples included in the thesis had no cap layer.

The simplest AlGaN/GaN HEMT structure consisting of AlGaN barrier on GaN bufferchannel layer grown on AlN/SiC templates (Fig. 1.14) was investigated in this thesis. The simplicity of the structure enabled the investigation of the pure effect of Al-content in the 


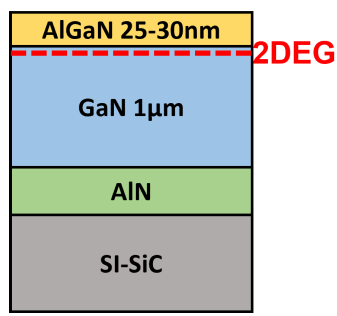

Figure 1.14: Schematic representation of the AlGaN/GaN HEMT structures. The top interface of the thick GaN buffer layer with the AlGaN barrier, serves as the channel area where the 2DEG is formatted.

barrier layer on the 2DEG properties, excluding any effects from the AlN interlayer or the GaN cap layer (Paper III).

\subsection{Characterization techniques}

\subsubsection{Optical Microscopy}

Optical microscopy is an efficient technique for getting quick feedback about the surface morphology and overall material quality of the samples. Imaging the sample using an optical microscope can reveal significant film non-uniformity, microscopic defects and any other microscopic surface features. Differential interference contrast microscopy Nomarski differential interference contrast (NDIC) is used to initially characterize all the samples included in this thesis, directly after growth. NDIC enhances the contrast along the edges of structural features making them more pronounced for easier observation. The working principle of NDIC relies on the phase shifting between two orthogonally polarised light beams upon their crossing through an optically transparent sample. Structural features in the sample, lead the two beams to follow a different optical path resulting in phase shifting. The recombination of the phase shifted beams results in interference and the optical result is the brightening or darkening of each point on the image. The high contrast Nomarski images reveal structural defects (e.g. pits, etched points, surface pyramids) that help for the tuning of the growth process parameters in order to maintain the desired quality in epitaxial layers. An example of optical microscopy images from $\mathrm{GaN}$ layers grown on $\mathrm{SiC}$ substrate is given in Fig. 1.15. 

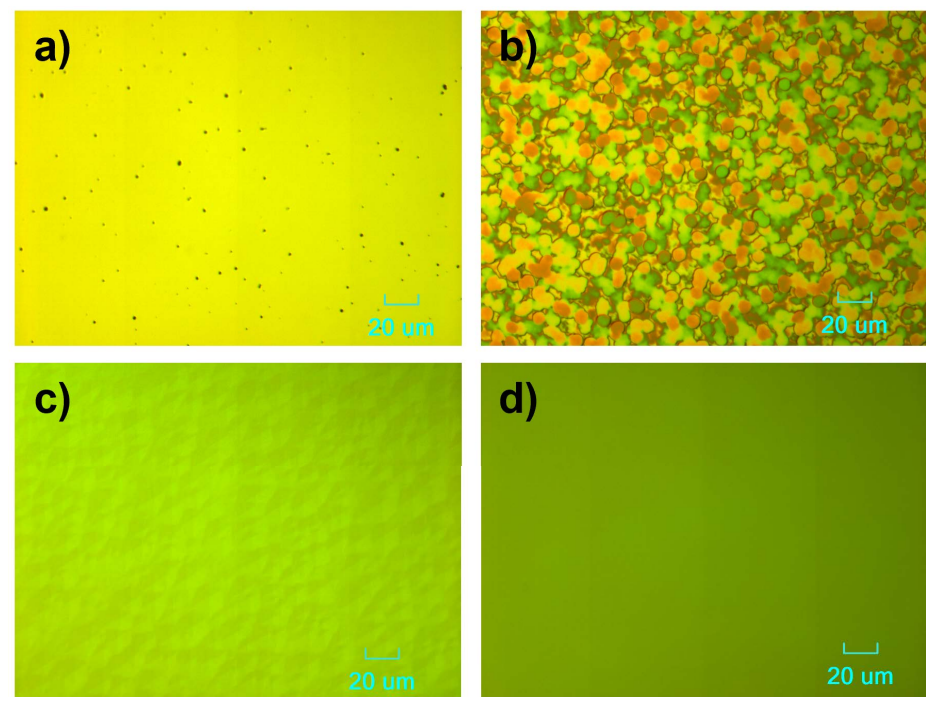

Figure 1.15: NDIC optical microscope images (at 400x magnification) of GaN epilayers showing different surface morphologies: $a$ ) pits, $b$ ) not continuous film, $c$ ) surface pyramidal features and $d$ ) flat GaN surface with no defects.

\subsubsection{Atomic force Microscopy}

A highly surface-sensitive technique widely used for imaging the surface topography of epilayers is atomic force microscopy (AFM) which is a type of scanning probe microscopy (SPM). AFM produces images with topography details in the nanometer scale by scanning the sample surface with a sharp tip (probe) and translating the sample-probe interaction to height, amplitude and phase images. For the AFM measurement, a sharp tip is scanned over the surface in a line-by-line manner. The tip (curvature radius of $\approx 10 \mathrm{~nm}$ ) is placed on a cantilever with reflective backside coating. The interaction forces between the probe and the surface atoms cause vertical and lateral deflections of the cantilever resulting in the displacement of a laser beam that is reflected on the backside of the cantilever. The laser spot displacement is sensored by a photodiode detector and the translated height signal forms the image. For the $\mathrm{x}-\mathrm{y}-\mathrm{z}$ - positioning of the probe, the scanning is done by a piezoelectric tube which allows for movement with precision in the nm-scale. A feedback correction loop for the driving signal is constantly adjusting the driving voltage on the piezotube. The AFM can be operated in three different modes: contact mode, non-contact mode and tapping mode. In contact mode the tip is scanning the surface 


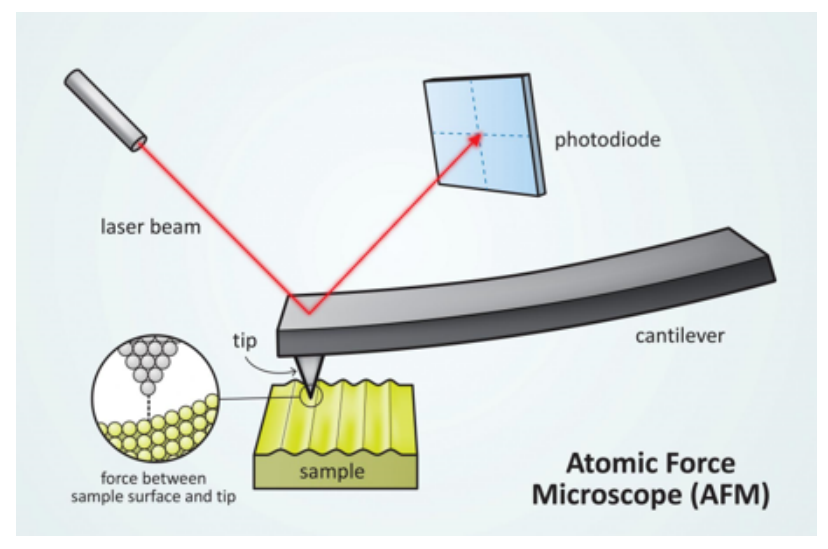

Figure 1.16: AFM working principle. Reprinted from Ref. [85]

while being in physical contact with it. In this case, the strong force interaction results in high spatial resolution but there is a high risk for tip-sample damage as well. In non-contact mode, the cantilever oscillates at a constant height above the surface, therefore the interaction is through long forces resulting in low spatial resolution in the imaging. Finally, in the hybrid (tapping) mode, the tip oscillates above the sample surface but it also lightly touches the surface at the lowest point of the oscillation. The tapping mode offers a high spatial resolution and a low risk for tip-sample damage, thus it is preferred and employed for all the AFM measurements included in the thesis. All the measurements presented in the thesis are performed using a Dimension 3100 instrument from Veeco, enabling vertical resolution of $\sim 0.3-1 \AA$ (limited by background noise). The lateral resolution very much depends on the tip radius of curvature $(\sim 10 \mathrm{~nm})$ and in our case is $\sim 1-5 \mathrm{~nm}$. The AFM provides fast imaging of the sample surface with details at the sub-nanometer scale and serves as a complementary technique to the NDIC. From the AFM images of III-nitride layers, surface defects like pits, etching spots, cracks, dislocations intersection with the surface, pyramids or hillocks are prominently revealed. Other important information relative to the growth conditions can be revealed e.g. the step size or the terraces dimensions. An example of AFM images of Mg-doped GaN is shown on Fig. 1.17.

\subsubsection{Spectroscopic ellipsometry}

Spectroscopic ellipsometry (SE) is a contactless non-destructive technique for probing the optical properties of layers by measuring frequency dependent changes in the polarization 

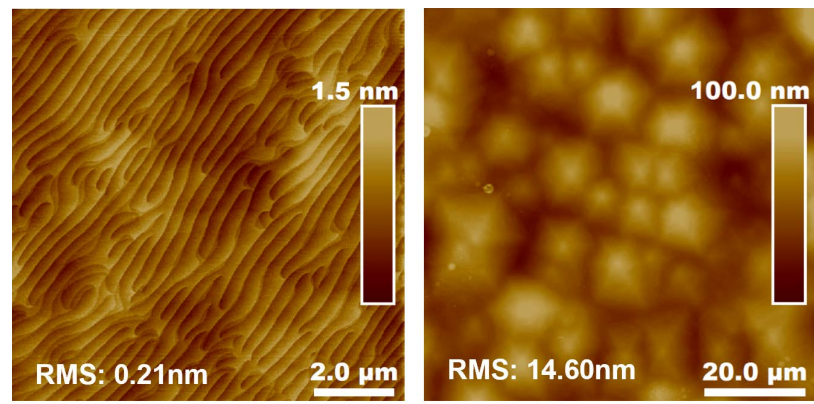

Figure 1.17: AFM images of Mg-doped GaN layers. Step-flow growth mode on lightly Mg-doped GaN (left) and formation of hexagonal pyramids on the surface of highly Mg-doped GaN (right).

state of light upon interaction with the sample material [86]. A simplified description of an ellipsometer setup includes the source lamp, a polarizer, the sample stage, the analyzer and the detector. The spectroscopic ellipsometry measurements included in this thesis were performed on a J. A. Woollam RC2-XI ellipsometer in the ultraviolet-visible spectral range $(0.7-5.9 \mathrm{eV})$. Since in $\mathrm{SE}$ the measured quantity is the ratio between the in-plane

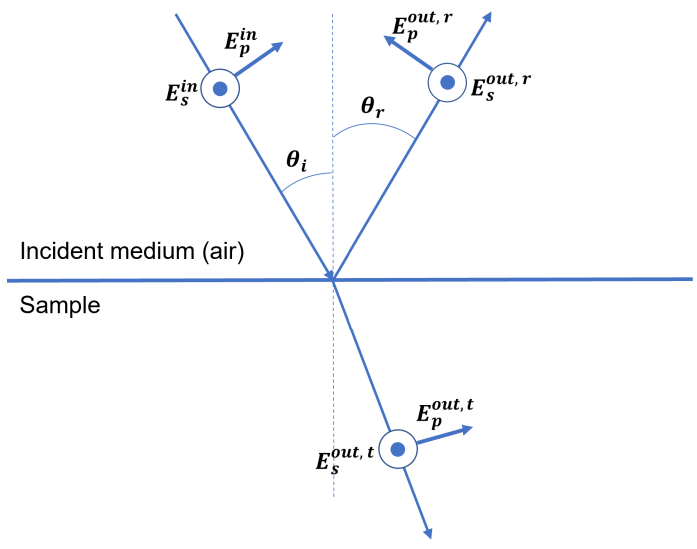

Figure 1.18: s- and p- polarized light components after reflection at a sample interface.

(p-) and out-of-plane (s-) polarized light and not the absolute intensity of reflected or transmitted light, the technique is insensitive to source light fluctuations, background radiation etc. In the case of light reflection from a sample, the ratio between the outcoming 
and incoming electric field for the s- and p-polarized light component is described by the complex reflection coefficients $r_{s}$ amd $r_{p}$ :

$$
r_{s}=\frac{E_{s}^{o u t}}{E_{s}^{\text {in }}} \quad r_{p}=\frac{E_{p}^{\text {out }}}{E_{p}^{\text {in }}}
$$

and the ratio between them is expressed as the ellipsometric data outcome:

$$
\rho=\frac{r_{p}}{r_{s}}=\tan (\Psi) \exp (i \Delta)
$$

For the determination of the ellipsometric parameters $\Psi$ and $\Delta$, the dielectric function $\varepsilon$ and the thickness of each of the layers should be taken into account and modelled properly. The best fitting parameter values will determine the $\Psi$ and $\Delta$.

In case of optically anisotropic samples, conversion between s- and the p-polarized light happens when the light is reflected from the sample. In this case, the previously described method of standard ellipsometry, determining $\Psi$ and $\Delta$ is not sufficient to fully describe the optical response of the sample. In this case, generalized ellipsometry approach should be used where the polarization state of light is represented by the Stokes vectors:

$$
\left(\begin{array}{c}
S_{1} \\
S_{2} \\
S_{3} \\
S_{4}
\end{array}\right)=\left(\begin{array}{c}
I_{p}+I_{s} \\
I_{p}-I_{s} \\
I_{+45}-I_{-45} \\
I_{R C}-I_{L C}
\end{array}\right)
$$

where: $I_{p}$ and $I_{s}$ are the intensities of the p- and s- polarized light, $I_{+45}$ and $I_{-45}$ are the intensities of the linearly polarized light rotated with respect to the plane of incidence by $+45^{\circ}$ and $-45^{\circ}$ respectively, and $I_{R C}$ and $I_{L C}$ are the intensities of the right-handed and left-handed circularly polarized light. The Stokes parameters are real-valued and the incident and reflected light Stokes vectors, $S^{\text {in }}$ and $S^{\text {out }}$ are connected via the $4 \times 4$ Mueller matrix:

$$
\left(\begin{array}{l}
S_{1}^{\text {out }} \\
S_{2}^{\text {out }} \\
S_{3}^{\text {out }} \\
S_{4}^{\text {out }}
\end{array}\right)=\left(\begin{array}{llll}
M_{11} & M_{12} & M_{13} & M_{14} \\
M_{21} & M_{22} & M_{23} & M_{24} \\
M_{31} & M_{32} & M_{33} & M_{34} \\
M_{41} & M_{42} & M_{43} & M_{44}
\end{array}\right)\left(\begin{array}{c}
S_{1}^{\text {in }} \\
S_{2}^{\text {in }} \\
S_{3}^{\text {in }} \\
S_{4}^{\text {in }}
\end{array}\right) .
$$

The Mueller matrix elements values are obtained by the ellipsometric measurement and contain all the information about the optical properties and thickness of the studied thin films. Using the appropriate model, one can fit the experimental data and acquire the optical constants and the thickness of each layer in a multi-layered structure. For optically 
in-plane isotropic samples, the off-diagonal elements of the Mueller matrix are equal to zero so, the matrix gets a more simplified form. In such case, the standard ellipsometry is sufficient for characterization of the sample. All the samples included in the current thesis are characterized using Mueller matrix ellipsometry, despite being optically isotropic, since any deviation from this behavior could only be identified using Mueller matrix ellipsometry.

\subsubsection{Optical Hall effect}

The OHE is an ellipsometry-based method which can be used for determination of the free charge carrier concentration, mobility and effective mass in conductive materials. The OHE describes the optical birefringence of conductive materials in the IR and $\mathrm{THz}$ frequency range. The optical birefringence occurs due to the motion of free charge carriers in the material under the influence of the Lorentz force, upon exposure of the sample to a magnetic field. This phenomenon is observed when the off-diagonal elements of the dielectric function tensor of the material are augmented by the magnetic field, which results in conversion between s- and p-polarized light upon reflection or transmission. The dielectric function tensor of conductive materials at the IR and THz range, when they are exposed to an external magnetic field is written as:

$$
\varepsilon=\varepsilon_{\infty}+\chi^{L}+\chi^{F C C-M O}
$$

where $\varepsilon_{\infty}$ is the magnetic field-independent high frequency tensor, $\chi^{L}$ is the lattice electric susceptibility tensor, and $\chi^{F C C-M O}$ is the magneto-optic free charge carrier electric susceptibility tensor. The magneto-optic electric susceptibility tensor, $\chi^{F C C-M O}$ is given by:

$$
\chi^{F C C-M O}=-\omega_{p}^{2}\left(\omega^{2} \mathbf{I}+i \omega \gamma_{p}-i \omega \omega_{c}\left(\begin{array}{ccc}
0 & -b_{z} & b_{y} \\
b_{z} & 0 & -b_{x} \\
-b_{y} & b_{x} & 0
\end{array}\right)\right)^{-1}
$$

where $\overrightarrow{\mathbf{B}}=|\overrightarrow{\mathbf{B}}|\left(b_{x}, b_{y}, b_{z}\right)$ is the magnetic field vector, $\mathbf{I}$ is the unit matrix and $\boldsymbol{\omega}_{c}=$ $q|\overrightarrow{\mathbf{B}}| \mathbf{m}_{\text {eff }^{-1}}$ is the cyclotron frequency tensor. For isotropic materials, the magneto-optic free charge carrier electric susceptibility $\chi^{F C C-M O}$ contains three independent parameters: the plasma frequency $\omega_{p}$, the plasma broadening $\gamma_{p}$ and the cyclotron frequency $\omega_{c}$. These parameters depend on the free charge carrier concentration $\mathrm{N}$, the mobility $\mu$ and the effective mass $\mathrm{m}_{\mathrm{eff}}$ parameters as shown in Eqns. 1.18, 1.19 and 1.20.

$$
\gamma_{p}=\frac{1}{\tau}=\frac{e}{\mu m_{\mathrm{eff}}} \rightarrow \mu=\frac{e \tau}{m_{\mathrm{eff}}}
$$




$$
\begin{aligned}
& \omega_{p}^{2}=\frac{N e^{2}}{m_{\mathrm{eff}} \varepsilon_{0}}, \\
& \omega_{c}=\frac{e|B|}{m_{\mathrm{eff}}},
\end{aligned}
$$

where $e$ is the unit charge and $\tau$ is the scattering time. Since the magnitude of the conversion among s- and p-polarized light due to the OHE strongly depends on the free charge carrier sheet density and mobility parameters, for samples with low sheet carrier density and mobility, the sensitivity of the OHE measurement is limited. A modification of the measurement method by adding a backside cavity with a highly reflective backside surface, cavity-enhanced OHE, enhances the OHE in HEMT structures, as a result of the formation of Fabri-Pérot oscillations within the sample-cavity system [87, 88]. Cavityenhanced THz OHE $[89,90]$ was employed for the 2DEG properties characterization in our samples. The measurements were carried out at RT under a magnetic field a field of $\pm 0.6 \mathrm{~T}, 45^{\circ}$ angle of incidence and an air gap of $\approx 100 \mu \mathrm{m}$ between the sample substrate and the surface of the magnet.

\subsubsection{High-resolution X-ray Diffraction}

High-resolution X-ray diffraction (HR-XRD) is commonly used for the characterization of the structural properties of epitaxial layers. It provides information on lattice parameters, alloy composition, strain, defect densities in the layers etc. Since HR-XRD is a non-destructive and fast characterization method, it is extensively employed for the evaluation of the structural quality in III-nitrides.

The wavelength of $\mathrm{x}$-rays is comparable to the interplanar spacing in crystals (few $\AA$ ) thus, allowing the characterization of their crystallographic structure. The incident $x$-rays radiation undergoes elastic scattering upon interaction with the core electrons of the atoms giving rise to diffraction effects. The result of the interference of the scattered $x$-rays from the sample is a diffraction pattern. A peak of intensity in the diffraction pattern corresponds to constructive interference from a set of (hkl) lattice planes when the Bragg's law condition is fulfilled:

$$
n \lambda=2 d \cdot \sin (\theta),
$$

where $n$ is the diffraction order, $\lambda$ is the $\mathrm{x}$-rays wavelength, $\theta$ is the angle of incidence and $d$ is the interplanar spacing between two adjacent planes, as illustrated in Fig. 1.19. Considering the wurtzite hexagonal crystal structure, the interplanar distance equation results in:

$$
d_{h k l}=\sqrt{\frac{1}{\frac{4}{3}\left(\frac{h^{2}+h k+k^{2}}{a^{2}}\right)+\frac{l^{2}}{c^{2}}}},
$$




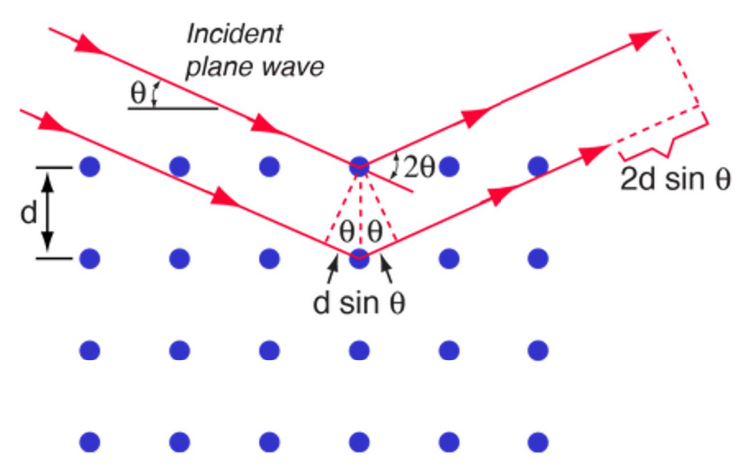

Figure 1.19: X-ray diffraction from crystal lattice planes with interplanar distance $d$.

where: $a$ and $c$ are the wurtzite lattice constants. In the wurtzite hexagonal crystal system, a four Miller index system is usually used in order to classify the family of planes, $\{h k i l\}$ with $i=-(h+k)$.

\subsubsection{Lattice parameters}

For the lattice constants determination, $2 \theta-\omega$ scans of at least one symmetric and one asymmetric peak must be measured. $\theta$ is the angle between the incident and the diffracted beam while, $\omega=\theta$ is the angle of incidence for the $x$-rays. Finite layer thickness and heterogeneous strain are the main factors causing broadening of the $2 \theta-\omega$ peaks. Using Eq.1.21 and Eq.1.22 for the measured $\theta$ of the symmetric peak, e. g. 0002, the $c$ lattice parameter can be determined. Repeating these steps for an asymmetric peak, e. g. 10 $\overline{1} 5$, and using the already determined $c$ lattice parameter, one can estimate the $a$ parameter.

For the HR-XRD structural characterization of the samples discussed in the current thesis, a PANalytical Empyrean diffractometer was used. At the incident x-ray beam, a hybrid monochromator consisting of a parabolic x-ray mirror and a two-bounce $\mathrm{Ge}(220)$ crystals provided x-rays with wavelength $\lambda=1.5405974 \AA$ (CuK $\alpha_{1}$ radiation) while, on the detector side, a symmetric three-bounce $\mathrm{Ge}(220)$ analyzer provided a $2 \theta$ resolution of $\sim 0.003^{\circ}(\sim 11 \mathrm{arcsec})$. The symmetric 0006 and asymmetric $10 \overline{1} 5 \mathrm{Bragg}$ reflections were used to determine the $c$ and $a$ lattice parameters, respectively.

After the $a$ and $c$ lattice parameters determination, the in-plane and out-of-plane strain values $\epsilon_{\mathrm{xx}}$ and $\epsilon_{\mathrm{zz}}$ can be determined by Eq. 1.23, where $\alpha_{0}$ and $c_{0}$ are the strain-free lattice parameters which can be obtained from the literature $[20,91]$. 


$$
\epsilon_{\mathrm{xx}} \equiv \frac{\alpha-\alpha_{0}}{\alpha_{0}} \quad \epsilon_{\mathrm{zz}} \equiv \frac{c-c_{0}}{c_{0}}
$$

In the case of lattice parameters determination, a RSM of an asymmetric peak can be used instead of the single $2 \theta-\omega$ scans. A reciprocal space map consists of sequential $2 \theta-\omega$ scans where the $\omega$ is varied by a small constant step for each scan. The measured data are then visualized on intensity contour plots. A lot of information can be extracted from the inensity, the position and the shape of the measured peak on a RSM, e. g. the lattice parameters, the crystalline quality and the residual strain in the layer. RSM of the asymmetric $10 \overline{1} 5$ and the symmetric 0004 peaks were measured for the Mg-doped GaN samples in order to identify effects related to the structural quality of the material after doping.

The crystalline quality of the layers can be also evaluated by measuring $\omega$-scans (rocking curves) of several symmetric and asymmetric peaks. For the $\omega$-scan, the detector is stationary while the sample is rocking around the $\omega$-axis. Assuming that the film is made of small crystals (mosaic block model) with slight misorientation between them, we can characterize them by the coherence length in horizontal and vertical direction, as well as by a tilt and twist angle, using the broadening of the $\omega$-scans.

\subsubsection{Dislocation density}

The screw- and edge- type dislocation densities, $N_{S}$ and $N_{E}$, were estimated by a commonly used method proposed by Srikant et al. [92, 93]. In order to use this method it is necessary to determine the tilt, $\alpha_{S}$, and twist, $\alpha_{E}$, angles and use the values of the Burgers vectors along the $c$ - and $a$-axis, $b_{c}$ and $b_{a}$, respectively.

$$
\begin{aligned}
& N_{S}=\frac{\alpha_{S}^{2}}{4.35 b_{c}^{2}} \\
& N_{E}=\frac{\alpha_{E}^{2}}{4.35 b_{a}^{2}}
\end{aligned}
$$

The tilt angle, $\alpha_{S}$, can be determined from the slope of the Williamson-Hall plot ( $\beta \sin \theta$ / $\lambda$ versus $\sin \theta / \lambda$ ) for the symmetric 0002, 0004, 0006 peaks of GaN [93]. Using $\alpha_{S}$ and the magnitude of the Burgers vector along the $c$-axis $\left(b_{c}=0.5185 \mathrm{~nm}\right)$ in Eq. 1.24, we estimate the screw-type dislocation density. For the estimation of edge dislocation density, the twist angle $\alpha_{E}$ is obtained from the fitting of the plot of integral widths $\beta$ of the rocking curves from the asymmetric $10 \overline{1} 1,10 \overline{1} 2,10 \overline{1} 3,10 \overline{1} 410 \overline{1} 5$, and $30 \overline{3} 2$ peaks, versus the inclination angle $\mathcal{X}$ (the clockwise angle between the diffraction vector normal and the sample surface) [92]. A fixed value for $\alpha_{S}$, which is obtained from the Williamson-Hall plot is used to fit 
the data and obtain $\alpha_{E}$. The Burgers vector along the $a$-axis with a value of $b_{a}=0.3189$ $\mathrm{nm}$ is used for the estimation of $N_{\mathrm{E}}$.

\subsubsection{Al composition in AlGaN}

The Al-content ( $\mathrm{x}$ ) determination in $\mathrm{Al}_{\mathrm{x}} \mathrm{Ga}_{1-\mathrm{x}} \mathrm{N}$ ternary alloys can be estimated from the lattice parameters of the respective layers [94]. The heteroepitaxial growth and the lattice mismatch between the GaN, AlGaN and AlN, result in large residual strain in the layers. Therefore, strain must be taken into account to avoid erroneous results. Under biaxial strain, $\epsilon_{\mathrm{xx}}=\epsilon_{\mathrm{yy}}$, the stress along the growth direction [0001], $\sigma_{\mathrm{zz}}$, is zero as the surface is free to expand or retract during growth or cooling down:

$$
\sigma_{\mathrm{zz}}=0=C_{13} \epsilon_{\mathrm{xx}}+C_{13} \epsilon_{\mathrm{yy}}+C_{33} \epsilon_{\mathrm{zz}},
$$

and since the in-plane strain is $\epsilon_{\mathrm{xx}}=\epsilon_{\mathrm{yy}}$, the Eq. 1.25 can be rewritten as:

$$
\epsilon_{\mathrm{zz}}=-\frac{2 C_{13}}{C_{33}} \epsilon_{\mathrm{xx}}
$$

where $C_{13}$ and $C_{33}$ are the elastic stiffness constants.

For the measured lattice parameters, $a(x)$ and $c(x)$, the Eq. 1.26 is formed as:

$$
\begin{array}{r}
\frac{c(x)-c_{0}(x)}{c_{0}(x)}=R_{B}(x) \frac{\alpha(x)-\alpha_{0}(x)}{\alpha_{0}(x)} \\
R_{B}(x)=-\frac{2 C_{13}(x)}{C_{33}(x)}
\end{array}
$$

where $\alpha_{0}(x), c_{0}(x)$ and $R_{B}(x)$ are the relaxed lattice parameters and the biaxial relaxation coefficient, respectively, for $\mathrm{AlGaN}$ with $\mathrm{Al}$-content -x. $C_{13}(x)$ and $C_{33}(x)$ are the elastic stiffness constants of $\mathrm{Al}_{\mathrm{x}} \mathrm{Ga}_{1-\mathrm{x}} \mathrm{N}$.

According to Vergard's rule, it is assumed that there is a linear dependency of the lattice parameters and the elastic stiffness constants with the Al-content, from 0 to $100 \%$ for GaN and AlN respectively:

$$
\begin{gathered}
\alpha_{0}(x)=x \alpha_{0}^{A l N}(x)+(1-x) \alpha_{0}^{G a N} \\
c_{0}(x)=x c_{0}^{A l N}(x)+(1-x) c_{0}^{G a N} \\
R_{B}(x)=x R_{B}^{A l N}+(1-x) R_{B}^{G a N}
\end{gathered}
$$

Eq. 1.27 can then be re-written as Eq.1.29 and the solution of this equation which lies in the range between $0 \leq x \leq 1$ gives the Al-content of the alloy. After determination of the 
Al-content $\mathrm{x}$, the relaxed lattice parameters $\alpha_{0}(x), c_{0}(x)$ and the strain can be determined by Eq. 1.28 and Eq. 1.23 respectively.

$$
2 C_{13}(x) \frac{\alpha-\alpha_{0}(x)}{\alpha_{0}(x)}+C_{33}(x) \frac{c-c_{0}(x)}{c_{0}(x)}=0
$$

\subsubsection{Mercury probe capacitance-voltage}

Capacitance-Voltage (C-V) measurement is a non-destructive method for determining the net carrier concentration (donor or acceptor) in a semiconductor layer as a function of thickness. The measurement requires the formation of two Schottky contacts which are realized by liquid mercury. The surface areas of the contacts differ a lot, with their diameters being $3 \mathrm{~mm}$ for the large and $0.7 \mathrm{~mm}$ for the small contact, respectively. The larger contact is set at forward bias and is considered as quasi-ohmic contact compared to the smaller Schottky contact. Thus, the free charge carriers below the Schottky contact can be depleted when the the contact is reverse biased. In addition to the DC bias, a small sinusoidal AC voltage $(\sim 100 \mathrm{mV})$ which is operating with frequency in the range of $100 \mathrm{~Hz}-1 \mathrm{MHz}$ is applied in order to change the width of the depletion layer. An LCR meter is measuring the depletion capacitance versus the bias voltage and from the measured data we can determine the net acceptor concentration $\left(N_{A}-N_{D}\right)$ in the semiconductor layer as a function of depth. The net doping concentration and the depletion depth are given by Eq.1.30 and Eq.1.31 respectively.

$$
\begin{gathered}
N_{A}-N_{D}=\frac{-C^{3}}{q A^{2} \varepsilon_{0} \varepsilon_{r}\left(\frac{d C}{d V}\right)}=\frac{2}{q A^{2} \varepsilon_{0} \varepsilon_{r} \frac{d\left(C^{-2}\right)}{d V}}, \\
x_{d}=\frac{A \varepsilon_{0} \varepsilon_{r}}{C},
\end{gathered}
$$

where $q$ is the elementary charge, $A$ is the Schottky contact area, $C$ is the capacitance, $x_{d}$ is the depletion region depth and $\varepsilon_{0}$ and $\varepsilon_{r}$ is the vacuum permittivity and the dielectric constant of the semiconductor, respectively. The net doping concentration is estimated from the slope of the plotted $C^{-2}-V$ curve using Eq. 1.30.

The $\mathrm{C}-\mathrm{V}$ measurement accuracy relies to a great extent on the precision in determination of the Schottky contact area. More importantly, in the case of p-type $\mathrm{Mg}$ dopants which are deep acceptors in GaN, the frequency of the test signal should be low enough so that all the electrically active acceptors have enough time to be ionized. The time constant $\left(\tau_{h}\right)$ for the holes emission to the valence band is given by Eq. 1.32, which depends on the hole capture cross section $\left(\sigma_{h}\right)$, the thermal velocity of holes $\left(v_{t h}\right)$, the effective density of states in the valence band at certain temperature $\left(N_{V}\right)$, and the energy gap between the valence 
band and the trap level $\left(E_{V}-E_{T}\right)$ i.e. the ionization energy of the acceptor. Using high frequencies of the test signal would result to partial ionization and underestimation of the electrically active $\mathrm{Mg}$ acceptors.

$$
\tau_{h}=\frac{\exp \left(E_{V}-E_{T}\right)}{\sigma_{h} v_{t h} N_{V}}
$$

It should be noted that for relatively high $\mathrm{Mg}$ concentrations $\sim 10^{19} \mathrm{~cm}^{-3}$, the depletion layer becomes very thin and consequently the tunneling through this layer increases. The increased leakage current in the Schottky diode leads to erroneous capacitance data thus, the useful range for extracting the net acceptor concentration relies only on a narrow voltage range.

For the determination of the net acceptor concentration in the Mg-doped GaN samples, the $\mathrm{C}-\mathrm{V}$ measurements were performed using a Hg-probe setup with a 4284A LCR meter from Agilent. The measurements were done in series measurement mode in the $1-10$ $\mathrm{kHz}$ frequency range with $\mathrm{AC}$ voltage of $100 \mathrm{mV}$.

In the case of AlGaN/GaN HEMT, C-V measurements allow us to determine the sheet carrier density of the 2DEG, $N_{\mathrm{s}}$, by integrating the area under the curve of volume charge distribution:

$$
N_{\mathrm{s}}=\int_{0}^{V_{\mathrm{R}}} n_{\mathrm{s}}(x) d V,
$$

where $n_{\mathrm{S}}(x)$ and $V_{\mathrm{R}}$ are the free charge carrier distribution and the reverse bias voltage respectively. As the reverse bias increase, the integrated charge carrier density increases and saturates at the pinch-off voltage, $\mathrm{V}_{\mathrm{p}}$, where all the free carriers are depleted. For further increase of $V_{R}$, there is no increase of the charge carrier density which is saturated at the $\mathrm{N}_{\mathrm{s}}$ value. Furthermore, the AlGaN barrier thickness $t$ (width of depletion region as in Eq.1.31), can be estimated by the slope of the Eq.1.33 plot for bias lower than the $V_{p}$ by assuming that the system resembles a parallel plate capacitor (Eq. 1.34).

$$
\begin{aligned}
C & =\frac{\Delta n_{\mathrm{s}}}{\Delta V}=\frac{\mathrm{A} \varepsilon_{0} \varepsilon_{\mathrm{r}}}{t} \\
t & =\mathrm{A} \varepsilon_{0} \varepsilon_{\mathrm{r}}\left(\frac{\Delta V}{\Delta n_{\mathrm{s}}}\right)
\end{aligned}
$$

\subsubsection{Electrical Hall effect measurement}

The electrical transport properties i.e. the type and the concentration of carriers as well as the mobility can be determined by Hall effect measurements. The measurement is based on the Hall voltage which is generated when the sample is subjected to an external 


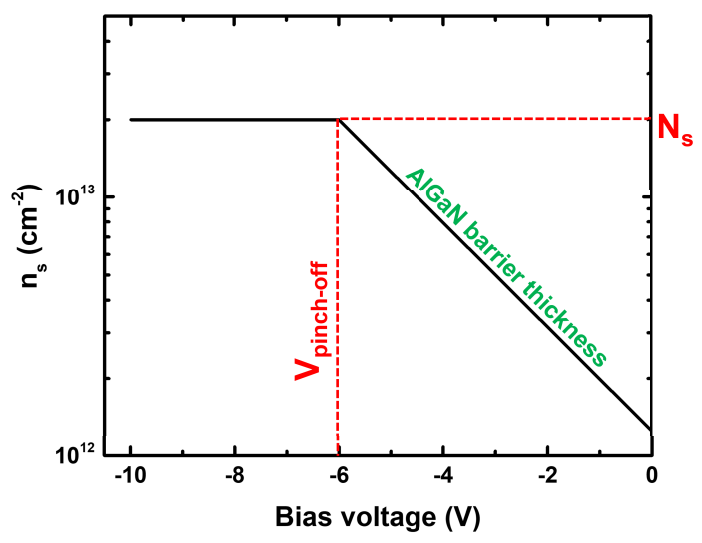

Figure 1.20: $\mathrm{N}_{\mathrm{s}}$ versus the depletion bias voltage

magnetic field $\mathbf{B}$ applied in perpendicular direction to the current flowing through the semiconductor. The Lorentz force which is induced by the magnetic field, makes the charge carriers to deflect from their original flow direction, causing a potential difference in perpendicular direction to the current. The calculated Hall coefficient, $R_{H}$ (Eq. 1.35) is then used for the determination of the carrier concentration (Eq.1.36), the mobility (Eq. 1.37) and the resistivity (Eq. 1.38).

$$
\begin{gathered}
R_{H-\text { electrons }}=\frac{1}{q n} \quad R_{H-\text { holes }}=\frac{1}{q p} \\
n \approx \frac{-I B}{q t V_{H}} \quad p \approx \frac{I B}{q t V_{H}} \\
\mu_{e} \approx \frac{1}{\rho q n} \quad \mu_{h} \approx \frac{1}{\rho q p} \\
\rho=\frac{1}{q\left(n \mu_{e}+p \mu_{h}\right)}
\end{gathered}
$$

In the equations above, $q$ is the elementary charge, $n$ or $p$ is the carrier concentration, $\mu_{e}$ or $\mu_{h}$ is the mobility of either electrons or holes, $t$ is the layer thickness and $\rho$ is the resistivity. The electrical properties of the samples studied in this thesis were characterized by Hall effect with either room temperature or temperature-dependent measurements. $\mathrm{Ni} / \mathrm{Au}(5 / 250 \mathrm{~nm})$ contacts were evaporated on the $\mathrm{p}-\mathrm{GaN}$ and annealed at $450{ }^{\circ} \mathrm{C}$ in air 
in order to become ohmic, and the samples were measured in Van der Pauw configuration using a Linseis HCS 1 instrument.

\subsubsection{Contactless measurements of electrical properties}

Post growth electrical characterization of the HEMT structures is necessary for the growth process optimization. For the electrical characterization, deposition of metal contacts with good ohmic behavior is required. Such post-growth processes are time consuming and invasive. Therefore, contactless methods are preferably used to simplify the measurement process and at the same time, they are not destructive. For the electrical characterization of the HEMT structures included in this thesis, we used contactless methods for measuring the sheet resistance and the sheet carrier density and mobility of the 2DEG. These measurements require a highly resistive substrate to avoid any interference from the charge carriers in the substrate thus, measuring only the contribution of the epilayers in the overall structure conductivity.

The sheet resistance $\left(R_{\mathrm{s}}\right)$ of the samples is measured by a method based on power consumption by the sample and creation of Eddy currents. An oscillating magnetic field is induced near the sample by an RF alternating current applied on coils. The coils are winded on a ferrite core which has a thin gap where the semiconductor material is inserted in. The sample is consequently under electromagnetic coupling with the instrument [95]. The $R_{S}$ can then be extracted by the power absorbed by the sample through Eq. 1.39.

$$
P_{\mathrm{s}}=\left(\frac{E_{\mathrm{T}}^{2}}{\left.8 \pi n^{2}\right)}\right) \sigma t \quad \text { and } \quad \sigma t=\frac{1}{\rho} t=\frac{1}{R_{\mathrm{s}}}
$$

where, $P_{\mathrm{S}}$ is the absorbed power assuming no flux leakage phenomena, $E_{\mathrm{T}}$ is the rms primary RF voltage, $n$ is the number of primary turns on the ferrite core. $\sigma, \rho$ and $t$ is the semiconductor conductivity, resistivity and thickness respectively. By applying this method we can measure the $R_{s}$. The instrument used for these measurements is an Eichhorn Hausmann MX604 with a working range of $50-3000$ ohms/square.

The 2DEG mobility and carrier density are measured by a microwave Hall-probe technique. A microwave signal $(10 \mathrm{GHz})$ is directed perpendicular to the HEMT structure surface through a circular waveguide. The sample is subjected to magnetic field of different strength and polarity in the range of $\pm 1 \mathrm{~T}$. The forward and reflected Hall power and its polarization is measured by the instrument and converted to the reflection coefficients used for the determination of the field-dependent conductivity tensors $\sigma_{X X}$ and $\sigma_{X Y}[96,97]$. 
The resistivity $\rho$, Hall coefficient $R_{H}$, sheet carrier density $N_{\mathrm{s}}$ and mobility $\mu$ can then be determined from equations Eq. 1.40, 1.41, 1.42 and 1.43.

$$
\begin{gathered}
\rho(\mathrm{H})=\frac{\sigma_{\mathrm{XX}}(\mathrm{H})}{\sigma_{\mathrm{XX}}^{2}(\mathrm{H})+\sigma_{\mathrm{XY}}^{2}(\mathrm{H})} \\
R_{\mathrm{H}}=\frac{-1}{H}\left(\frac{\sigma_{X X}(\mathrm{H})}{\sigma_{X X}^{2}(\mathrm{H})+\sigma_{\mathrm{XY}}^{2}(\mathrm{H})}\right) \\
N_{\mathrm{s}}=\frac{-1}{e R_{\mathrm{H}}} \\
\mu=\frac{-R_{\mathrm{H}}}{\rho(\mathrm{H})}
\end{gathered}
$$

A single-carrier or multi-carrier model can be considered for determining the 2DEG transport properties either for a single type of carriers or a multi-carrier system. For the latter, the contribution of each carrier in the overall transport behavior is presented in terms of carrier density and mobility. Since the transport properties are extracted form fitting, defining as many parameters as possible to the algorithm will result in more accurate fitting outcome. Therefore, the $R_{S}$ and the epilayer thickness are provided as input for the measurement. $R_{s}$ is measured by the contactless method described before while the thickness is measured by spectroscopic ellipsometry.

\subsubsection{Secondary ion mass spectroscopy}

Secondary ion mass spectroscopy (SIMS) is a very useful technique for quantification of the impurity concentrations, namely $\mathrm{H}, \mathrm{O}, \mathrm{Si}$ and $\mathrm{C}$, as well as the $\mathrm{Mg}$ doping concentration in GaN. Since the impurities affect the electrical properties of the material, it is crucial to minimize them by tuning of the growth parameters and SIMS provides the quantitative data that are necessary for doing so. Furthermore, the Mg concentration profile in the as-grown $\mathrm{GaN}$ and the difference of $\mathrm{H}$-level in as-grown and annealed $\mathrm{GaN}$ are of great importance since they are directly related to the electrical properties of the material. For these reasons, SIMS was extensively used for the characterization of samples studied in this thesis. Additionally, SIMS measurement on a sample grown with reference conditions, can reveal possible changes of the growth chamber conditions and probably explain any 
drifting in material quality over time. SIMS uses a focussed ion beam of Cs to sputter

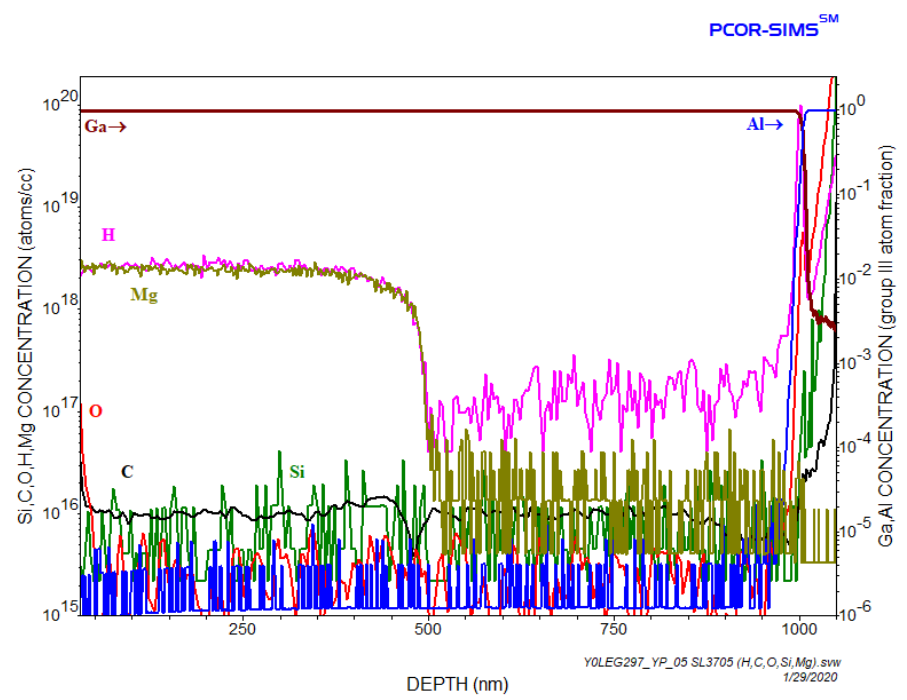

Figure 1.21: SIMS measurement of a Mg-doped GaN sample

the layers under investigation. The charged secondary ions extracted from the layer are collected and analyzed by mass spectrometers for element identification and quantification over depth. The resolution of SIMS depends on the sputtering rate which is chosen depending on the epilayer thickness. All the measurements were performed by Evans Analytical Group laboratories with depth resolution of $10 \mathrm{~nm}$. The detection limit for the various elements were typically the same for any measurement: $\mathrm{H}: 3-5 \times 10^{16} \mathrm{~cm}^{-3}, \mathrm{C}$ and O: $1-2 \times 10^{15} \mathrm{~cm}^{-3}$, Si: $2 \times 10^{15} \mathrm{~cm}^{-3}$ and Mg: $1-2 \times 10^{16} \mathrm{~cm}^{-3}$. An example of a SIMS measurement from a GaN:Mg/UID-GaN/AlN/SiC is shown on Fig. 1.21.

\subsection{Summary of main results}

The main focus of this thesis is the development of $p$-GaN growth by hot-wall MOCVD. The goal is to achieve state-of-the-art $\mathrm{Mg}$ doping and activation in $\mathrm{GaN}$ in order to implement $p$-GaN in power devices. To achieve this goal, we explored hot-wal MOCVD for $p$-type GaN growth. We have performed a systematic investigation of the effects of growth conditions on the incorporation of $\mathrm{Mg}, \mathrm{H}$ and $\mathrm{C}$ in the as-grown GaN:Mg. Several growth parameters like the $\mathrm{V} / \mathrm{III}$ ratio, pressure, temperature, $\mathrm{Cp} 2 \mathrm{Mg} / \mathrm{TMGa}$ ratio and carrier 
gas mixture of $\mathrm{H}_{2}$ and $\mathrm{N}_{2}$ were varied for the experiments. After initial optimization of growth conditions UID- and GaN:Mg with low background impurity concentrations of [O] and [Si] below the SIMS detection limit of $(2-5) \times 10^{15} \mathrm{~cm}^{-3}$, and $[\mathrm{C}]<10^{16} \mathrm{~cm}^{-3}$ are demonstrated. This allows for further optimization of $\mathrm{Mg}$ incorporation and free hole properties. The $[\mathrm{C}]$ is greatly affected by the growth conditions and can be tuned by its almost linear dependence on Ga-supersaturation (Paper I).

We have shown that the $\mathrm{Mg}$ concentration in the range $\sim 2.5 \times 10^{18}-1.1 \times 10^{20} \mathrm{~cm}^{-3}$ scales linearly with the Mg-to-Ga precursor flux ratio but it is also affected to some extent by the Ga-supersaturation. $[\mathrm{H}]$ has been shown to closely follow [Mg] up to $\approx 2.4 \times 10^{19}$ $\mathrm{cm}^{-3}$. However, in contrast to earlier reports, we find that $[\mathrm{H}]$ may be up to $22 \%$ lower than the typically observed 1:1 ratio with $[\mathrm{Mg}]$ resulting in $p$-type conductivity of as-grown GaN:Mg. The net acceptor concentration $N_{\mathrm{A}}-N_{\mathrm{D}}$ after annealing of GaN:Mg, follows the $[\mathrm{Mg}]$ in this range of concentrations (up to $2.4 \times 10^{19} \mathrm{~cm}^{-3}$ ) showing that all the $\mathrm{Mg}$ atoms are incorporated in the $\mathrm{Mg}_{\mathrm{Ga}}$ (acceptor) site. Based on these results a process for efficient $p$-type doping with a resistivity of $0.77 \Omega$.cm and a free hole concentration of $8.4 \times 10^{17}$ $\mathrm{cm}^{-3}$ corresponding to $\mathrm{Mg}$ acceptor activation of $5.25 \%$ has been established (Paper I).

For $[\mathrm{Mg}] \geq 2.4 \times 10^{19} \mathrm{~cm}^{-3},[\mathrm{H}]$ deviates significantly from the $1: 1$ ratio with up to $84 \%$ lower $[\mathrm{H}]$. In this range of $\mathrm{Mg}$ concentrations, $N_{\mathrm{A}}-N_{\mathrm{D}}$ and the respective free-holes concentrations are considerably lower than $[\mathrm{Mg}]$ implying formation of Mg-related defects in GaN:Mg (Paper I). Indeed, we have shown that the structural quality of GaN:Mg layers is deteriorated as revealed by the increase of threading dislocation densities and the generation of Mg-related defects in the form of PIDs where the Mg segregates. This is accompanied by the formation of large hexagonal hillocks which increase in height and density with $[\mathrm{Mg}]$ increase, as revealed by AFM images (Fig. 1.22) (Paper I). STEM analysis further has shown that the segregated $\mathrm{Mg}$ is sufficient to account for the observed amount of electrically inactive $\mathrm{Mg}$ not bound to $\mathrm{H}$. However, generation of $\mathrm{V}_{\mathrm{N}}$ and its complexes with $\mathrm{Mg}_{\mathrm{Ga}}$ need to be invoked to explain the considerably lower free hole and net acceptor concentrations measured in the highly doped GaN:Mg grown at high supersaturation.

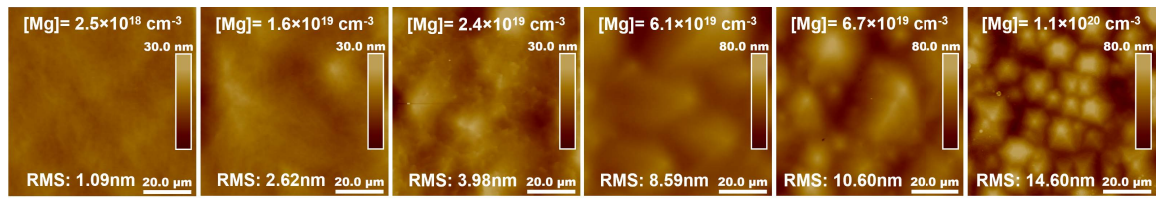

Figure 1.22: Mg-doped GaN with increasing $[\mathrm{Mg}]$ from $2.5 \times 10^{18}-1.1 \times 10^{20} \mathrm{~cm}^{-3}$. Hexagonal pyramids are form on the surface with increasing height and density upon increase of incorporated $\mathrm{Mg}$ level. 
We further focused our efforts on increasing the amount of non-passivated $\mathrm{Mg}$ acceptors in as-grown GaN:Mg below the threshold of PID formation by using high amounts of $\mathrm{H}_{2}$ in the carrier gas mixture during growth. We have shown that using this approach one can increase by more than one order of magnitude the free hole concentration in as-grown GaN:Mg, e.g., from $7.6 \times 10^{15}$ to $8.6 \times 10^{16} \mathrm{~cm}^{-3}$ ) for material grown under 'regular' and high- $\mathrm{H}_{2}$ growth environment, respectively (Paper II). The use of $1 \mu \mathrm{m}$ thick $\mathrm{GaN}$ buffer layer to reduce the dislocation density allows for further enhancement of free hole concentration in as-grown GaN:Mg. Overall, the optimized structure sample grown under high- $\mathrm{H}_{2}$ environment exhibited free-holes concentration of $2.0 \times 10^{17}$ and $9.8 \times 10^{17} \mathrm{~cm}^{-3}$ and resistivity of 3.7 and $0.7 \Omega \cdot \mathrm{cm}$, for as-grown and post-annealing condition, respectively (Paper II). These results and the established comprehensive picture of GaN:Mg growth via hot-wall MOCVD substantiate the expanded capabilities of the technique to deliver state-of-the-art $p$-type GaN for the development of power diodes and transistors (Paper I and Paper II).

Additionally, in this thesis we have dedicated efforts to the polarization doping of GaN in order to realize high-frequency HEMT device structures with improved linearity. As a first step we have investigated the 2DEG properties in AlGaN/GaN HEMTs by THzOHE measurements combined with structural characterization and simulations. We have studied a series of $\mathrm{AlGaN} / \mathrm{GaN}$ HEMT structures with varying Al-content of the AlGaN barrier in the range $7-42 \%$. The structures have smooth surface morphology $(<0.4 \mathrm{~nm}$ rms roughness) which is an indication of a low channel/barrier interface roughness. The 2DEG density is found to increase with the Al-content, as expected but, the mobility peaks at $\sim 20 \%$ of $\mathrm{Al}$ and decreases for higher $\mathrm{Al}$ percentage. For the high Al-content barriers, the 2DEG effective mass, $\mathrm{m}_{\mathrm{eff}}$, is found to decrease while for the lower $\mathrm{Al} \%$ the $\mathrm{m}_{\mathrm{eff}}$ is increased, contrary to what one would expect. The 2DEG mobility decreases for the low Al-content barrier layers because of the higher $\mathrm{m}_{\mathrm{eff}}$ while for high Al-content barriers this is due to the decreasing scattering time (paper III). The results presents a good basis for the next steps in realizing high-Al content AlGaN/GaN HEMTs needed for high-frequency applications and for the growth of HEMT structures with improved linearity. 


\section{References}

[1] D. Ueda, in Power GaN Devices (Springer, 2017), pp. 1-26.

[2] M. Kanechika, T. Uesugi, and T. Kachi, in 2010 international electron devices meeting (IEEE, 2010) , pp. 13-5.

[3] J.-G. Kim, C. Cho, E. Kim, J. S. Hwang, K.-H. Park, and J.-H. Lee, IEEE Trans. Electron Devices 68, 1513 (2021).

[4] F. Medjdoub, N. Herbecq, A. Linge, and M. Zegaoui, in 2015 IEEE International Electron Devices Meeting (IEDM) (IEEE, 2015) , pp. 9-2.

[5] H. Xing, Y. Dora, A. Chini, S. Heikman, S. Keller, and U. Mishra, IEEE Electron Device Letters 25, 161 (2004).

[6] J.-T. Chen, J. Bergsten, J. Lu, E. Janzén, M. Thorsell, L. Hultman, N. Rorsman, and O. Kordina, Appl. Phys. Lett. 113, 041605 (2018).

[7] T. Nakamura, in Workshop on IoT/M2M and Future Radio Access (2012).

[8] Y. Mancuso, P. Gremillet, and P. Lacomme, in 2006 IEEE MTT-S International Microwave Symposium Digest (IEEE, 2006) , pp. 614-617.

[9] H. Hommel and H. Feldle, in First European Radar Conference, 2004. EURAD. (IEEE, 2004), pp. 121-124.

[10] B. Chu, B. Kang, H. Wang, C. Chang, Y. Tseng, A. Goh, A. Sciullo, W. Wu, J. Lin, B. Gila, et al., in Gallium Nitride Materials and Devices IV (International Society for Optics and Photonics, 2009), vol. 7216p. 72162A.

[11] Y. Zhong, J. Zhang, S. Wu, L. Jia, X. Yang, Y. Liu, Y. Zhang, and Q. Sun, Fundamental Research (2021). 
[12] S. Nakamura, Reviews of Modern Physics 87, 1139 (2015).

[13] Z. Mi and C. Jagadish, III-Nitride Semiconductor Optoelectronics, (Academic Press, 2017).

[14] C. Zhou, A. Ghods, V. G. Saravade, P. V. Patel, K. L. Yunghans, C. Ferguson, Y. Feng, B. Kucukgok, N. Lu, and I. T. Ferguson, ECS Journal of Solid State Science and Technology 6, Q149 (2017).

[15] R. Zwierz, Plasma enhanced growth of GaN single crystalline layers from vapour phase, $\mathrm{PhD}$ thesis, (2014).

[16] H. Morkoç, Handbook of nitride semiconductors and devices, Materials Properties, Physics and Growth, vol. 1, (John Wiley \& Sons, 2009).

[17] J. Wu, J. Appl. Phys. 106, 5 (2009).

[18] F. Bernardini, V. Fiorentini, and D. Vanderbilt, Physical Review B 56, R10024 (1997).

[19] F. Morales, J. Mánuel, R. García, B. Reuters, H. Kalisch, and A. Vescan, Journal of Physics D: Applied Physics 46, 245502 (2013).

[20] V. Darakchieva, B. Monemar, and A. Usui, Appl. Phys. Lett. 91, 031911 (2007).

[21] F. Morales, D. González, J. Lozano, R. García, S. Hauguth-Frank, V. Lebedev, V. Cimalla, and O. Ambacher, Acta Materialia 57, 5681 (2009).

[22] O. Ambacher, J. Smart, J. Shealy, N. Weimann, K. Chu, M. Murphy, W. Schaff, L. Eastman, R. Dimitrov, L. Wittmer, et al., J. Appl. Phys. 85, 3222 (1999).

[23] O. Ambacher, B. Foutz, J. Smart, J. Shealy, N. Weimann, K. Chu, M. Murphy, A. Sierakowski, W. Schaff, L. Eastman, et al., J. Appl. Phys. 87, 334 (2000).

[24] S. Sharbati, I. Gharibshahian, T. Ebel, A. A. Orouji, and W.-T. Franke, Journal of Electronic Materials 50, 3923 (2021).

[25] I. Smorchkova, L. Chen, T. Mates, L. Shen, S. Heikman, B. Moran, S. Keller, S. DenBaars, J. Speck, and U. Mishra, J. Appl. Phys. 9o, 5196 (2001).

[26] C. Wood and D. Jena, Polarization effects in semiconductors: from ab initio theory to device applications, (Springer Science \& Business Media, 2007).

[27] D. Jena, in Polarization effects in semiconductors (Springer, 2008), pp. 161-216.

[28] L. Hsu and W. Walukiewicz, Physical Review B 56, 1520 (1997). 
[29] L. Hsu and W. Walukiewicz, J. Appl. Phys. 89, 1783 (2001).

[30] A. Gold, Physical Review B 35, 723 (1987).

[31] J.-T. Chen, I. Persson, D. Nilsson, C.-W. Hsu, J. Palisaitis, U. Forsberg, P. O. Persson, and E. Janzén, Appl. Phys. Lett. 106, 251601 (2015).

[32] M. Meneghini, G. Meneghesso, and E. Zanoni, Cham: Springer International Publishing (2017).

[33] G. Miceli and A. Pasquarello, Physical Review B 93, 165207 (2016).

[34] J. Lyons, A. Janotti, and C. Van de Walle, J. Appl. Phys. 115, 012014 (2014).

[35] C. G. Van de Walle and J. Neugebauer, J. Appl. Phys. 95, 3851 (2004).

[36] P. Vennéguès, M. Leroux, S. Dalmasso, M. Benaissa, P. De Mierry, P. Lorenzini, B. Damilano, B. Beaumont, J. Massies, and P. Gibart, Physical Review B 68, 235214 (2003)

[37] R. F. Pierret and G. W. Neudeck, Advanced semiconductor fundamentals, vol. 6, (AddisonWesley Reading, MA, 1987).

[38] A. Kasic, M. Schubert, J. Off, B. Kuhn, F. Scholz, S. Einfeldt, T. Böttcher, D. Hommel, D. J. As, U. Köhler, A. Dadgar, A. Krost, et al., physica status solidi (c) o, 1750(2003).

[39] J. L. Lyons, A. Alkauskas, A. Janotti, and C. G. Van de Walle, physica status solidi (b) 252, 900 (2015).

[40] V. Ramachandran, R. Feenstra, W. Sarney, L. Salamanca-Riba, J. Northrup, L. Romano, and D. Greve, Appl. Phys. Lett. 75, 808 (1999).

[41] P. Tavernier, T. Margalith, J. Williams, D. Green, S. Keller, S. DenBaars, U. Mishra, S. Nakamura, and D. Clarke, Journal of crystal growth 264, 150 (2004).

[42] N. Fichtenbaum, C. Schaake, T. Mates, C. Cobb, S. Keller, S. DenBaars, and U. Mishra, Appl. Phys. Lett. 91, 172105 (2007).

[43] B. Beaumont, S. Haffouz, and P. Gibart, Appl. Phys. Lett. 72, 921 (1998).

[44] J. E. Northrup, Appl. Phys. Lett. 82, 2278(2003).

[45] T. Narita, K. Tomita, Y. Tokuda, T. Kogiso, M. Horita, and T. Kachi, J. Appl. Phys. 124, 215701 (2018). 
[46] T. Narita, K. Tomita, K. Kataoka, Y. Tokuda, T. Kogiso, H. Yoshida, N. Ikarashi, K. Iwata, M. Nagao, N. Sawada, M. Horita, J. Suda, and T. Kachi, Jpn. J. Appl. Phys. 59, $\mathrm{SAo804}$ (2020).

[47] K. Iwata, T. Narita, M. Nagao, K. Tomita, K. Kataoka, T. Kachi, and N. Ikarashi, Applied Physics Express 12, 031004 (2019).

[48] T. Remmele, M. Albrecht, K. Irmscher, R. Fornari, and M. Straßburg, Appl. Phys. Lett. 99, 141913 (2011).

[49] Q. Yan, A. Janotti, M. Scheffler, and C. G. Van de Walle, Appl. Phys. Lett. 100, 142110 (2012).

[5o] J. Yang, D. G. Zhao, D. S. Jiang, P. Chen, Z. S. Liu, L. C. Le, X. J. Li, X. G. He, J. P. Liu, S. M. Zhang, H. Wang, J. J. Zhu, and H. Yang, Journal of Applied Physics 115, 163704(2014).

[51] S. Liu, J. Yang, D. Zhao, D. Jiang, F. Liang, P. Chen, J. Zhu, Z. Liu, X. Li, W. Liu, L. Zhang, H. Long, and M. Li, Superlattices and Microstructures 104, 63(2017).

[52] H. Xing, D. S. Green, H. Yu, T. Mates, P. Kozodoy, S. Keller, S. P. DenBaars, and U. K. Mishra, Jpn. J. Appl. Phys. 42, 50 (2003).

[53] A. C. Jones and M. L. Hitchman, Chemical vapour deposition: precursors, processes and applications, (Royal society of chemistry, 2009).

[54] P. Capper, S. Irvine, and T. Joyce, in Springer Handbook of Electronic and Photonic Materials (Springer, 2017), pp. 1-1.

[55] D. Koleske, A. Wickenden, R. Henry, and M. Twigg, Journal of crystal growth 242, 55 (2002).

[56] G. B. Stringfellow, Organometallic vapor-phase epitaxy: theory and practice, (Elsevier, 1999).

[57] S. Mita, R. Collazo, A. Rice, R. Dalmau, and Z. Sitar, J. Appl. Phys. 104, 013521 (2008).

[58] O. Kordina, C. Hallin, A. Ellison, A. S. Bakin, I. G. Ivanov, A. Henry, R. Yakimova, M. Touminen, A. Vehanen, and E. Janzén, Applied Physics Letters 69, 1456(1996).

[59] O. Kordina, C. Hallin, A. Henry, J. P. Bergman, I. Ivanov, A. Ellison, N. T. Son, and E. Janzén, physica status solidi (b) 202, 321(1997). 
[6o] A. Kakanakova-Georgieva, R. R. Ciechonski, U. Forsberg, A. Lundskog, and E. Janzén, Crystal Growth \& Design 9, 880(2009).

[61] J.-T. Chen, J. W. Pomeroy, N. Rorsman, C. Xia, C. Virojanadara, U. Forsberg, M. Kuball, and E. Janzén, Journal of Crystal Growth 428, 54 (2015).

[62] Z. C. Feng, III-Nitride semiconductor materials, (World Scientific, 2006).

[63] A. Kakanakova-Georgieva, U. Forsberg, I. Ivanov, and E. Janzén, Journal of Crystal Growth 300, 100 (2007), first International Symposium on Growth of Nitrides.

[64] J. Lu, J.-T. Chen, M. Dahlqvist, R. Kabouche, F. Medjdoub, J. Rosen, O. Kordina, and L. Hultman, Applied Physics Letters 115, 221601 (2019).

[65] N. T. Son, C. P. Anderson, A. Bourassa, K. C. Miao, C. Babin, M. Widmann, M. Niethammer, J. Ul Hassan, N. Morioka, I. G. Ivanov, F. Kaiser, J. Wrachtrup, and D. D. Awschalom, Applied Physics Letters 116, 190501(2020).

[66] V. Cimalla, J. Pezoldt, and O. Ambacher, Journal of Physics D: Applied Physics 4o, 6386 (2007).

[67] L. Liu and J. H. Edgar, Materials Science and Engineering: R: Reports 37, 61 (2002).

[68] T. Kimoto and J. A. Cooper, (2014).

[69] K. Inaba, Rigaku Journal 30 (2014).

[70] C. Hallin, F. Owman, P. Mårtensson, A. Ellison, A. Konstantinov, O. Kordina, and E. Janzén, Journal of crystal growth 181, 241 (1997).

[71] J. Hassan, J. Bergman, A. Henry, and E. Janzén, Journal of crystal growth 310, 4430 (2008).

[72] D. Koleske, R. Henry, M. Twigg, J. Culbertson, S. Binari, A. Wickenden, and M. Fatemi, Appl. Phys. Lett. 80, 4372 (2002).

[73] P. Waltereit, O. Brandt, A. Trampert, M. Ramsteiner, M. Reiche, M. Qi, and K. Ploog, Appl. Phys. Lett. 74, 3660 (1999).

[74] K. Jeganathan, M. Shimizu, and H. Okumura, Appl. Phys. Lett. 86, 073106 (2005).

[75] T.-C. Wen, S.-C. Lee, W.-I. Lee, T.-Y. Chen, S.-H. Chan, and J.-S. Tsang, Jpn. J. Appl. Phys. 40, L495 (2001). 
[76] S. Chung, J. Park, S. Kim, T. Wang, and S. Sakai, Journal of the Korean Physical Society 39, 499 (2001).

[77] B. Hull, S. Mohney, H. Venugopalan, and J. Ramer, Appl. Phys. Lett. 76, 2271 (2000).

[78] J. Lyons, A. Janotti, and C. Van de Walle, Appl. Phys. Lett. 97, 152108 (2010).

[79] J. L. Lyons, D. Wickramaratne, and C. G. Van de Walle, J. Appl. Phys. 129, 111101 (2021).

[8o] B. M. Green, K. K. Chu, E. M. Chumbes, J. A. Smart, J. R. Shealy, and L. F. Eastman, IEEE Electron Device Letters 21, 268 (2000).

[81] Z. Feng, Y. G. Zhou, S. Cai, and K. M. Lau, Appl. Phys. Lett. 85, 5248 (2004).

[82] R. Gutt, M. Himmerlich, M. Fenske, S. Müller, T. Lim, L. Kirste, P. Waltereit, K. Köhler, S. Krischok, and T. Fladung, J. Appl. Phys. 110, 083527 (2011).

[83] D. Marcon, F. Medjdoub, D. Visalli, M. Van Hove, J. Derluyn, J. Das, S. Degroote, M. Leys, K. Cheng, S. Decoutere, et al., in 2010 IEEE International Reliability Physics Symposium (IEEE, 2010), pp. 146-151.

[84] M. Charles, Y. Baines, R. Bouis, and A.-M. Papon, physica status solidi (b) 255, 1700406 (2018).

[85] nisenet.org/catalog/scientific-image-atomic-force-microscope illustration, .

[86] H. Fujiwara, Spectroscopic ellipsometry: principles and applications, (John Wiley \& Sons, 2007).

[87] S. Knight, S. Schöche, V. Darakchieva, P. Kühne, J.-F. Carlin, N. Grandjean, C. M. Herzinger, M. Schubert, and T. Hofmann, Opt. Lett. 40, 2688(2015).

[88] N. Armakavicius, J.-T. Chen, T. Hofmann, S. Knight, P. Kühne, D. Nilsson, U. Forsberg, E. Janzén, and V. Darakchieva, physica status solidi c 13, 369(2016).

[89] M. Schubert, P. Kühne, V. Darakchieva, and T. Hofmann, J. Opt. Soc. Am. A 33, 1553(2016).

[9o] P. Kühne, N. Armakavicius, V. Stanishev, C. M. Herzinger, M. Schubert, and V. Darakchieva, IEEE Trans. Terahertz Sci. Technol. 8, 257(2018).

[91] D. Nilsson, E. Janzén, and A. Kakanakova-Georgieva, Journal of Physics D: Applied Physics 49, 175108(2016). 
[92] V. Srikant, J. Speck, and D. Clarke, J. Appl. Phys. 82, 4286 (1997).

[93] T. Metzger, R. Höpler, E. Born, O. Ambacher, M. Stutzmann, R. Stömmer, M. Schuster, H. Göbel, S. Christiansen, M. Albrecht, et al., Philosophical magazine A 77, 1013 (1998).

[94] F. Oehler, M. E. Vickers, M. J. Kappers, C. J. Humphreys, and R. A. Oliver, Japanese Journal of Applied Physics 52, o8JB29(2013).

[95] G. Miller, D. Robinson, and J. Wiley, Review of Scientific Instruments 47, 799 (1976).

[96] W. Beck and J. Anderson, J. Appl. Phys. 62, 541 (1987).

[97] D. Nguyen, K. Hogan, A. Blew, and M. Cordes, Journal of crystal growth 272, 59 (2004). 



\section{List of abbreviations}

2DEG two-dimensional electron gas

2DHG two-dimensional hole gas

AFM atomic force microscopy

Al aluminum

AlGaN aluminum gallium nitride

AlN aluminum nitride

Be beryllium

BV breakdown voltage

C carbon

CMP chemical mechanical polished

$\mathrm{Cp}_{2} \mathrm{Fe}$ bis(cyclopentadienyl) iron

$\mathrm{Cp}_{2} \mathbf{M g}$ bis(cyclopentadienyl) magnesium

C-V Capacitance-Voltage

$\mathbf{E}_{\mathrm{F}}$ Fermi level

Fe iron

G Gibbs free energy

Ga gallium

GaN gallium nitride

Ge germanium

GFR gas-foil-rotation

$\mathbf{H}$ hydrogen 
$\mathbf{H}_{2}$ hydrogen

hcp hexagonal close packed

HEMT high electron mobility transistor

HF high frequency

HP high power

HR-XRD High-resolution X-ray diffraction

In indium

InN indium nitride

IR infrared

LED light-emitting diode

MBE molecular beam epitaxy

$\mathbf{m}_{\text {eff }}$ effective mass

MFC mass flow controller

Mg magnesium

MO metal-organic

MOCVD metal-organic chemical vapor deposition

$\mathbf{N}$ nitrogen

$\mathbf{N}_{2}$ nitrogen

NDIC Nomarski differential interference contrast

$\mathbf{N H}_{3}$ ammonia

O oxygen

OHE optical Hall effect

$\mathbf{P}_{\mathrm{PE}}$ piezoelectric polarization

PIDs pyramidal inversion domains

$\mathbf{P}_{\mathrm{SP}}$ spontaneous polarization

$\mathbf{R F}$ radio frequency

$\mathbf{R}_{\mathrm{S}}$ sheet resistance

RSM reciprocal space map

RTA rapid thermal annealing

SE Spectroscopic ellipsometry 
Se selenium

Si silicon

SiC silicon carbide

SIMS Secondary ion mass spectroscopy

$\mathbf{S i}_{3} \mathbf{N}_{4}$ silicon nitride

SPM scanning probe microscopy

TaC tantalum carbide

TDs threading dislocations

TMAl trimethylaluminum

TMGa trimethylgallium

UID- unintentionally doped

UV ultraviolet 



\section{Part II}

\subsection{Publications included in the thesis}

\section{Paper I}

Alexis Papamichail, Anelia Kakanakova, Einar Ö. Sveinbjörnsson, Axel R. Persson, Björn Hult, Niklas Rorsman, Vallery Stanishev, Son P. Le, Per O. Å. Persson, Muhammad Nawaz, Jr-Tai Chen, Plamen P. Paskov, and Vanya Darakchieva "Mg-doping and free-hole properties of hot-wall MOCVD GaN" Submitted to Journal of Applied Physics

Contribution: I have been involved in the experimental design and I have performed all epitaxial growth runs. I also performed all AFM, XRD, C-V and SE thickness measurements and data analysis. I was involved in the Hall effect and TEM analysis and interpretation. I wrote the first draft of the manuscript.

\section{Paper II}

Anelia Kakanakova, Alexis Papamichail, Vallery Stanishev, and Vanya Darakchieva "Incorporation of magnesium into GaN regulated by intentionally large amounts of hydrogen in MOCVD processes"

Submitted to Applied Physics Letters

Contribution: I have been involved in the experimental design and I have performed all epitaxial growth runs. I also performed the AFM and SE thickness measurements and all data analysis and I was involved in the interpretation of the results and the manuscript writing.

\section{Paper III}


Steffen Richter, Alexis Papamichail, Philipp kühne, Shiqi Guo, Sean Knight, Axel R. Persson, Vallery Stanishev, Plamen P. Paskov, and Vanya Darakchieva

"2DEG properties of AlGaN/GaN HEMT structures with varying Al content in the AlGaN barrier layer probed by $\mathrm{THz}$ optical Hall effect"

In draft manuscript

Contribution: I have been involved in the experimental design and I have performed all epitaxial growth runs. I also performed all AFM, XRD, C-V, SE thickness measurements and contactless resistivity measurements and data analysis. I was involved in the interpretation of the results and writing of the manuscript.

\subsection{Publications not included in the thesis}

\section{Paper I}

Rafal Korlacki, Megan Stokey, Alyssa Mock, Sean Knight, Alexis Papamichail, Vanya Darakchieva, and Mathias Schubert

"Strain and stress relationships for optical phonon modes in monoclinic crystals with $\beta-G a_{2} \mathrm{O}_{3}$ as an example"

Phys. Rev. B 102, 180101(R) (2020)

\section{Paper II}

Eva Bittrich, Jari Domke, Dieter Jehnichen, Lars Bittrich, Mikhail Malanin, Andreas Janke, Petra Uhlmann, Klaus-Jochen Eichhorn, Alexis Papamichail, Vallery Stanishev, Vanya Darakchieva, Mahmoud Al-Hussein, Marieta Levichkova, Torsten Fritz, and Karsten Walzer "Morphology of Thin Films of Aromatic Ellagic Acid and Its Hydrogen Bonding Interactions"

J. Phys. Chem. C 2020, 124, 30, 16381־16390

\section{Paper III}

Hengfang Zhang, Ingemar Persson, Alexis Papamichail, Jr.-Tai Chen, Per O. Å. Persson, Plamen P. Paskov, and Vanya Darakchieva

"On the polarity determination and polarity inversion in nitrogen-polar group III-nitride layers grown on $\mathrm{SiC}^{\circ}$

J. Appl. Phys. 131, 055701 (2022) 


\section{Papers}

The papers associated with this thesis have been removed for copyright reasons. For more details about these see:

https://doi.org/10.3384/9789179292522 


\section{FACULTY OF SCIENCE AND ENGINEERING}

Linköping Studies in Science and Technology, Licentiate Thesis No. 1929, 2022 Department of Physics, Chemistry and Biology (IFM)

Linköping University

SE-581 83 Linköping, Sweden

www.liu.se 